\title{
Nonlinear Dynamics of Imperfect FGM Conical Panel
}

\author{
Yan Niu $\mathbb{D},{ }^{1}$ Yuxin Hao $\mathbb{D},^{2}$ Minghui Yao $\mathbb{D},{ }^{1}$ Wei Zhang $\mathbb{D},{ }^{1}$ and Shaowu Yang $\mathbb{D}^{1}$ \\ ${ }^{1}$ College of Mechanical Engineering, Beijing University of Technology, Beijing Key Laboratory of Nonlinear Vibrations and Strength of \\ Mechanical Structures, Beijing 100124, China \\ ${ }^{2}$ College of Mechanical Engineering, Beijing Information Science and Technology University, Beijing 100192, China
}

Correspondence should be addressed to Minghui Yao; merry_mingming@163.com

Received 7 August 2017; Accepted 26 November 2017; Published 27 February 2018

Academic Editor: M. I. Herreros

Copyright (C) 2018 Yan Niu et al. This is an open access article distributed under the Creative Commons Attribution License, which permits unrestricted use, distribution, and reproduction in any medium, provided the original work is properly cited.

\begin{abstract}
Structures composed of functionally graded materials (FGM) can satisfy many rigorous requisitions in engineering application. In this paper, the nonlinear dynamics of a simply supported FGM conical panel with different forms of initial imperfections are investigated. The conical panel is subjected to the simple harmonic excitation along the radial direction and the parametric excitation in the meridian direction. The small initial geometric imperfection of the conical panel is expressed by the form of the Cosine functions. According to a power-law distribution, the effective material properties are assumed to be graded along the thickness direction. Based on the first-order shear deformation theory and von Karman type nonlinear geometric relationship, the nonlinear equations of motion are established by using the Hamilton principle. The nonlinear partial differential governing equations are truncated by Galerkin method to obtain the ordinary differential equations along the radial displacement. The effects of imperfection types, half-wave numbers of the imperfection, amplitudes of the imperfection, and damping on the dynamic behaviors are studied by numerical simulation. Maximum Lyapunov exponents, bifurcation diagrams, time histories, phase portraits, and Poincare maps are obtained to show the dynamic responses of the system.
\end{abstract}

\section{Introduction}

Structures composed of functionally graded materials (FGM) offer more potential advantages than conventional composites and can satisfy many rigorous requisitions in engineering application. Because of their continuous graded variation of material properties along one direction of the structures, functionally graded materials have drawn great attention. Especially when functionally graded materials structures are made from metal and ceramic materials, they will have heat resistance capacity and high tensile strength. The FGM is beneficial to achieve desirable mechanical properties by changing the volume fraction index. Conical panels with different initial curvatures and geometric parameters are usually found in kinds of engineering applications, such as turbine blade and aircraft fuselage. Conical panels are often subjected to the axial load, the transverse load, and the thermal stress, which cause the complex nonlinear dynamic behaviors of the system. But, nonlinear dynamic analysis of the functionally graded materials conical panels has been paid less attention and investigation in the current literature.

Much research has been focused on the static analysis of the FGM conical panel. Aghdam et al. [1] investigated the bending of functionally graded material conical panels with the moderately thick under the clamped boundary condition. The panel was subjected to uniform and nonuniform distributed load, respectively. Abediokhchi et al. [2] considered various boundary conditions and analyzed the bending of FGM conical panels under the transverse load. They used generalized differential quadrature method to study the deflection of the panel with different volume fractions and geometric parameters. Aghdam et al. [3] applied the extended Kantorovich method to gain the accurate solution for the bending of the moderately thick FGM sector plate with all edges clamped. Duc et al. [4] discussed the linear stability of functionally graded material conical panel subjected to the thermal and the mechanical loads on elastic foundations. The conical panel and the stiffeners were deformed in thermal 
environments. Viola et al. [5] made a static analysis of FGM conical shells and panels.

Recently, the study on dynamics of the FGM conical panels has been given more attention. Most of investigations are focused on the free vibration and linear vibration of the FGM conical panels. Bardell et al. [6] performed experimental analysis of the sandwich conical panel and applied the finite element method to study vibration of the system. Tornabene and Viola [7] used the generalized differential quadrature method to study the dynamics of the moderately thick FGM parabolic panels and shells. Zhao and Liew [8] investigated free vibration of FGM conical panels by elementfree kp-Ritz method and analyzed the effect of geometrical and physical parameters on frequency characteristic. Akbari et al. [9] took account of the effects of parameters on natural frequencies and utilized generalized differential quadrature method to study free vibration of Levy type of FGM conical panels. Su et al. [10, 11] examined free vibration of FGM cylindrical, conical shells and annular plates by the RayleighRitz method. Xie et al. [12] explored a simple efficient solution for the free vibration analysis of functionally graded conical shells and annular plates. Tornabene [13] focused on the dynamic characters of the FGM conical, cylindrical shells, and annular plates.

Hafeez et al. [14] studied the buckling behavior of the geometric imperfect conical tanks subjected to the hydrostatic pressure. Maali et al. [15] discussed the buckling of simply supported thin conical panels with the imperfection. Zhao and Liew [16] gave a thermal-mechanical buckling analysis of FGM conical panels applying the element-free $\mathrm{kp}$-Ritz method. Bich et al. [17] investigated the linear buckling of FGM conical panel subjected to the axial and the external pressure. Javanbakht et al. [18] solved the dynamic equations for the simply supported FGM shallow and nonshallow shell by Galerkin and Newmark method.

It is well known that functionally graded materials are usually produced at the high temperature and are cooled with different methods. Thus, the initial geometric imperfections often occur in the FGM structures $[19,20]$. There are many studies on the buckling and the vibration analysis of the FGM plates and shells with geometrical imperfections. Huang and Han [21] studied the buckling behaviors of the imperfect functionally graded cylindrical shells under the axial compression. Sofiyev [22] analyzed the nonlinear prebuckling deformations and the initial imperfections, the nonlinear buckling of the functionally graded truncated conical shells subjected to an axial compressive excitation. Shariyat and Asgari [23] examined the nonlinear thermal buckling and postbuckling behaviors of the imperfect variable thickness cylindrical functionally graded shells. Samsam Shariat and Eslami [24] analyzed the thermal buckling of the imperfect rectangular functionally graded plates. Yang and Huang [25] investigated the nonlinear transient response for the simple supported imperfect functionally graded plates subjected to the thermal stress. Kitipornchai et al. [26] analyzed nonlinear vibration of laminated FGM plate with imperfection. Duc and Quan [27] took advantage of the Bubnov-Galerkin method to canvass the nonlinear response of the eccentrically stiffened functionally graded material cylindrical panels subjected to the mechanical loads. Liu et al. [28] investigated nonlinear vibration of the imperfect FGM cylindrical shells under the in-plane excitation and the transverse excitation.

However, few studies have been carried out on the dynamics of the functionally graded material conical panel with the initial imperfection. This paper is based on the work of our previous conference paper [29]. In this paper, the nonlinear dynamics of a simply supported FGM conical panel with different forms of the initial imperfections are investigated. The conical panel is subjected to the simple harmonic excitation along the radial direction and the parametric excitation in the meridian direction. The small initial geometric imperfection of the conical panel is expressed by the form of the Cosine functions. According to a powerlaw distribution, the effective material properties are assumed to be graded along the thickness direction. Based on the first-order shear deformation theory and von Karman type nonlinear geometric relationship, the nonlinear equations of motion are established by using the Hamilton principle. The nonlinear partial differential governing equations are truncated by Galerkin method to obtain the ordinary differential equations along the radial displacement. The effects of imperfection types, half-wave numbers of the imperfection, amplitudes of the imperfection, and damping on the dynamic behaviors are studied by numerical simulation. Maximum Lyapunov exponents, bifurcation diagrams, time histories, phase portraits, and Poincare maps are obtained to show the dynamic responses.

\section{Formulation}

2.1. The Model of the Imperfect FGM Conical Panel. A ceramic-metal FGM conical panel with initial geometric imperfection is considered, which has semivertex angle $\beta$, subtended angle $\gamma$, slanted length $L$, thickness $h$, and the radii $R_{1}$ and $R_{2}$ at the two ends, as shown in Figure 1. The curvilinear coordinate system $(x, \theta, z)$ is located on the midsurface of the conical panel, which is along the meridional, circumferential, and radial directions, respectively. It is assumed that the conical panel is subjected to the radial excitation $F(x, \theta) \cos \Omega_{1} t$ and the in-plane parametric excitation $p_{0}+$ $p_{1} \cos \left(\Omega_{2} t\right)$ at $x=0$ and $x=L$, respectively. Since any cyclical loading can be expressed as the series of simple harmonic series, a geometric imperfection shape of the conical panel in the radial direction is defined by $w^{*}$ and it can be expressed as follows $[25,26]$ :

$$
\begin{aligned}
& w^{*}=\eta h \sin \left(\frac{\delta_{1} \pi x}{L}\right) \sin \left(\frac{\delta_{2} \pi \theta}{\gamma}\right), \\
& w^{*}=\eta h \sin \left(\frac{\delta_{1} \pi x}{L}\right),
\end{aligned}
$$

where $\eta h$ indicates the amplitude of imperfection, $\delta_{1}$ and $\delta_{2}$ are half-wave numbers of the imperfection in the $x$ and $\theta$ axes, respectively, $w^{*}=\eta h \sin \left(\delta_{1} \pi x / L\right) \sin \left(\delta_{2} \pi \theta / \gamma\right)$ represents the imperfection including both the $x$ and $\theta$ axes, and $w^{*}=$ $\eta h \sin \left(\delta_{1} \pi x / L\right)$ is the imperfection only in $x$ axes. 


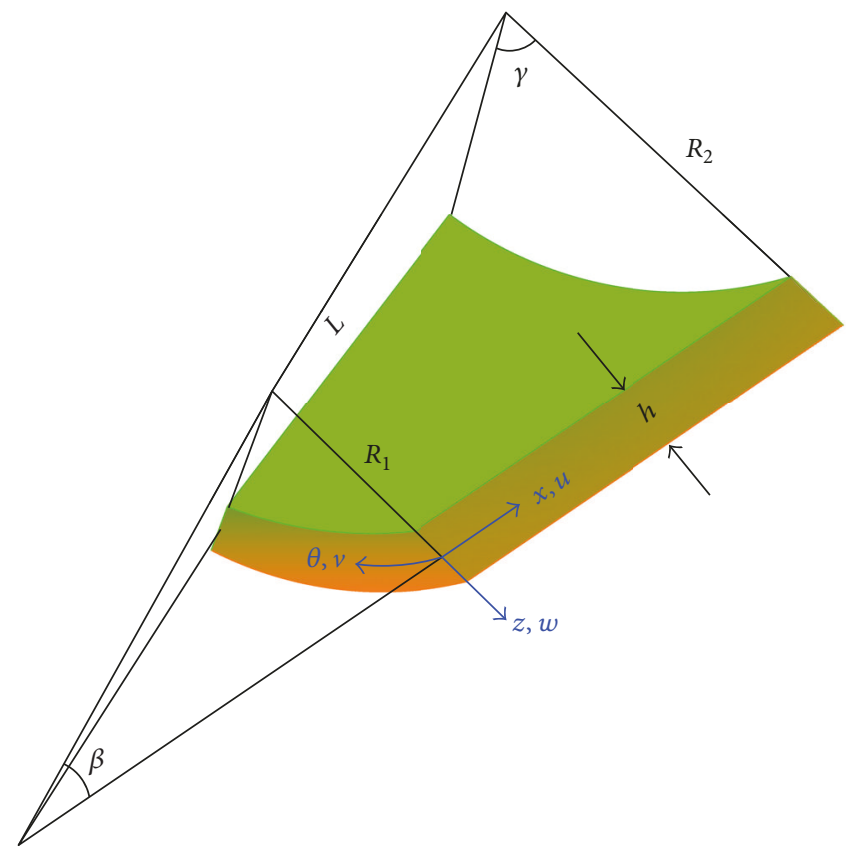

FIGURE 1: The model of the imperfect FGM conical panel is given.

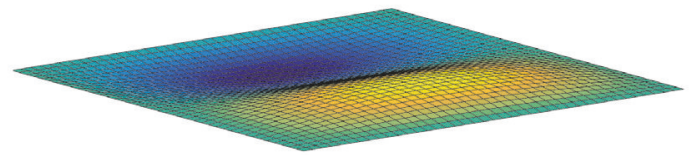

(a)

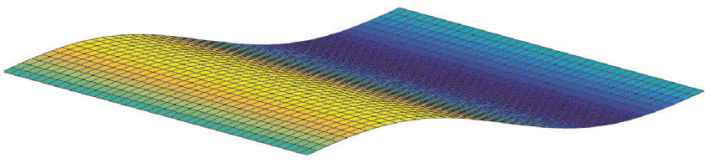

(c)

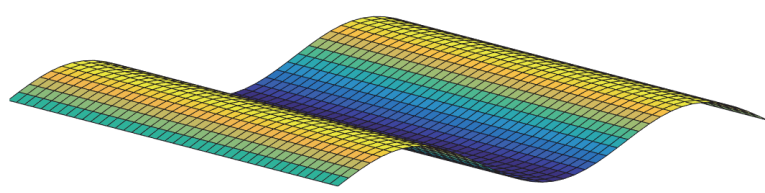

(e)

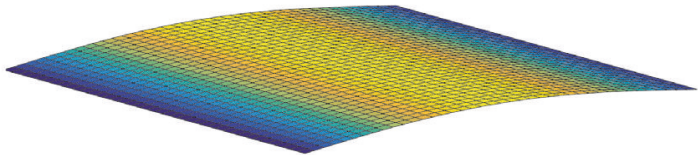

(b)

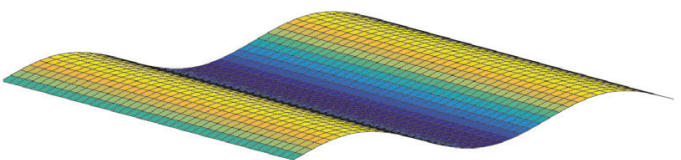

(d)

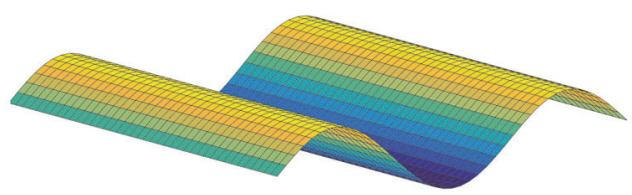

(f)

Figure 2: The imperfection types of the structure: (a) $0.1 h \sin (\pi x / L) \sin (2 \pi \theta / \gamma)$; (b) $0.1 h \sin (\pi x / L) ;$ (c) $0.1 h \sin (2 \pi x / L)$; (d) $0.1 h \sin (3 \pi x / L)$; (e) $0.2 h \sin (3 \pi x / L)$; (f) $0.3 h \sin (3 \pi x / L)$.

When the semivertex angle is $\beta=0$ and the radii at the two ends are $R_{1}=R_{2}=\infty$, the conical panel is simplified as a rectangle plate. Figure 2 shows the imperfection types of the structure investigated in this paper. Figure 2(a) describes the imperfection type of the structure, which is expressed by $0.1 h \sin (\pi x / L) \sin (2 \pi \theta / \gamma)$. Figure $2(\mathrm{~b})$ demonstrates the imperfection, which is represented by $0.1 h \sin (\pi x / L)$. Figure 2(c) displays the imperfection, which is defined by $0.1 h \sin (2 \pi x / L)$. Figures $2(\mathrm{~d})-2(\mathrm{f})$ show the imperfections, which are described as $0.1 h \sin (3 \pi x / L), 0.2 h \sin (3 \pi x / L)$, and $0.3 h \sin (3 \pi x / L)$, respectively.
The material properties are assumed to be varied continuously and smoothly in the thickness direction according to the power-law distribution. The volume fraction of the ceramic and the metal can be written as follows:

$$
\begin{gathered}
V_{c}(z)=\left(\frac{1}{2}+\frac{z}{h}\right)^{k}, \\
V_{m}(z)=1-V_{c}(z),
\end{gathered}
$$

where $k$ is the volume fraction index and subscripts $c$ and $m$ represent the ceramic and the metal, respectively. 
The effective material properties, such as Young's modulus $E$, mass density $\rho$, Poisson's ratio $\nu$, and the thermal expansion coefficient $\alpha$ can be obtained as follows:

$$
\begin{aligned}
& E=E_{c} V_{c}+E_{m} V_{m}, \\
& \rho=\rho_{c} V_{c}+\rho_{m} V_{m}, \\
& \nu=\nu_{c} V_{c}+\nu_{m} V_{m}, \\
& \alpha=\alpha_{c} V_{c}+\alpha_{m} V_{m} .
\end{aligned}
$$

The properties of the temperature dependent on materials are expressed by [30]

$$
P_{i}=P_{0}\left(P_{-1} T^{-1}+1+P_{1} T+P_{2} T^{2}+P_{3} T^{3}\right), \quad i=c, m,
$$

where $P_{0}, P_{-1}, P_{1}, P_{2}$, and $P_{3}$ are the coefficients of the temperature $T$ expressed in the form of Kelvin, and these coefficients are different for the different constituent materials.

2.2. Displacement and Constitutive Equations. Generally, dynamic response of the conical panel can be expressed in terms of displacements. According to first-order shear deformation theory, the displacement field of the imperfect FGM conical panel is assumed to be

$$
\begin{gathered}
u(x, \theta, z, t)=u_{0}(x, \theta, t)+z \phi_{x}(x, \theta, t), \\
v(x, \theta, z, t)=v_{0}(x, \theta, t)+z \phi_{\theta}(x, \theta, t), \\
w(x, \theta, z, t)=w_{0}(x, \theta, t)+w^{*}(x, \theta),
\end{gathered}
$$

where $\left(u_{0}, v_{0}, w_{0}\right)$ is the displacement of an arbitrary point on the middle surface of the conical panel in the coordinate system $(x, \theta, z), \phi_{x}$ is the rotation of the transverse normal about the $\theta$-axis, and $\phi_{\theta}$ denotes the rotation of the transverse normal about the $x$-axis.

According to the theory of von Karman large deformation, the relationships between strains and displacements are defined as follows:

$$
\left\{\begin{array}{l}
\varepsilon_{x x} \\
\varepsilon_{\theta \theta} \\
\gamma_{x \theta} \\
\gamma_{\theta z} \\
\gamma_{x z}
\end{array}\right\}=\left\{\begin{array}{l}
\varepsilon_{x x}{ }^{(0)} \\
\varepsilon_{\theta \theta}{ }^{(0)} \\
\gamma_{x \theta}{ }^{(0)} \\
\gamma_{\theta z}{ }^{(0)} \\
\gamma_{x z}{ }^{(0)}
\end{array}\right\}+z\left\{\begin{array}{l}
\varepsilon_{x x}{ }^{(1)} \\
\varepsilon_{\theta \theta}{ }^{(1)} \\
\gamma_{x \theta}{ }^{(1)} \\
\gamma_{\theta z}{ }^{(1)} \\
\gamma_{x z}{ }^{(1)}
\end{array}\right\}
$$

where

$$
\begin{aligned}
& \left\{\begin{array}{l}
\varepsilon_{x x}{ }^{(0)} \\
\varepsilon_{\theta \theta}{ }^{(0)} \\
\gamma_{x \theta}{ }^{(0)} \\
\gamma_{\theta z^{(0)}}{ }^{(0)}
\end{array}\right\}=\left\{\begin{array}{c}
\frac{\partial u_{0}}{\partial x}+\frac{1}{2}\left(\frac{\partial w_{0}}{\partial x}\right)^{2}+\frac{\partial w_{0}}{\partial x} \frac{\partial w^{*}}{\partial x} \\
\gamma_{x z} \frac{1}{R} \frac{\partial v_{0}}{\partial \theta}+\frac{1}{R} w_{0} \cos \beta+\frac{1}{R} w^{*} \cos \beta+\frac{1}{R} u_{0} \sin \beta+\frac{1}{2} \frac{1}{R^{2}}\left(\frac{\partial w_{0}}{\partial \theta}\right)^{2}+\frac{1}{R^{2}} \frac{\partial w_{0}}{\partial \theta} \frac{\partial w^{*}}{\partial \theta} \\
\frac{1}{R} \frac{\partial u_{0}}{\partial \theta}-\frac{1}{R} v_{0} \sin \beta+\frac{\partial v_{0}}{\partial x}+\frac{1}{R} \frac{\partial w_{0}}{\partial x} \frac{\partial w_{0}}{\partial \theta}+\frac{1}{R} \frac{\partial w_{0}}{\partial x} \frac{\partial w^{*}}{\partial \theta}+\frac{1}{R} \frac{\partial w^{*}}{\partial x} \frac{\partial w_{0}}{\partial \theta} \\
\phi_{\theta}+\frac{1}{R} \frac{\partial w_{0}}{\frac{\partial \theta}{\partial w_{0}}+\frac{1}{R} \frac{\partial w^{*}}{\partial \theta}-\frac{1}{R} v_{0} \cos \beta} \\
\frac{\partial w^{*}}{\partial x}+\frac{\phi_{x}}{\partial x}+\phi_{x}
\end{array}\right\}, \\
& \left\{\begin{array}{l}
\varepsilon_{x x}{ }^{(1)} \\
\varepsilon_{\theta \theta}{ }^{(1)} \\
\gamma_{x \theta}{ }^{(1)} \\
\gamma_{\theta z}{ }^{(1)} \\
\gamma_{x z}{ }^{(1)}
\end{array}\right\}=\left\{\begin{array}{c}
\frac{\partial \phi_{x}}{\partial x} \\
\frac{1}{R} \frac{\partial \phi_{\theta}}{\partial \theta}+\frac{1}{R} \phi_{x} \sin \beta \\
\frac{\partial}{R} \frac{\partial \phi_{x}}{\partial \theta}-\frac{1}{R} \phi_{\theta} \sin \beta+\frac{\partial \phi_{\theta}}{\partial x} \\
0 \\
0
\end{array}\right\} .
\end{aligned}
$$

The constitutive relations of the imperfect FGM conical panel considering the thermal stress are given by

$$
\left\{\begin{array}{c}
\sigma_{x} \\
\sigma_{\theta} \\
\sigma_{x \theta} \\
\sigma_{\theta z} \\
\sigma_{x z}
\end{array}\right\}=\left[\begin{array}{ccccc}
Q_{11} & Q_{12} & 0 & 0 & 0 \\
Q_{12} & Q_{22} & 0 & 0 & 0 \\
0 & 0 & Q_{66} & 0 & 0 \\
0 & 0 & 0 & Q_{44} & 0 \\
0 & 0 & 0 & 0 & Q_{55}
\end{array}\right]\left\{\begin{array}{l}
\varepsilon_{x x} \\
\varepsilon_{\theta \theta} \\
\gamma_{x \theta} \\
\gamma_{\theta z} \\
\gamma_{x z}
\end{array}\right\}
$$

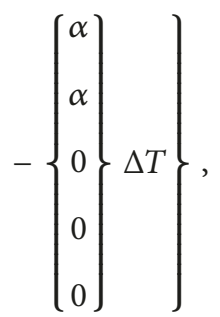


where

$$
\begin{aligned}
Q_{11} & =Q_{22}=\frac{E}{1-v^{2}}, \\
Q_{12} & =\frac{v E}{1-\nu^{2}}, \\
Q_{44} & =Q_{55}=Q_{66}=\frac{E}{2(1+\nu)} .
\end{aligned}
$$

2.3. Governing Equations. According to Hamilton's principle, the partial differential equation of motion can be derived as follows:

$$
\begin{aligned}
& N_{x x, x}+\frac{1}{R} N_{x \theta, \theta}+\frac{1}{R} N_{x x} \sin \beta-\frac{1}{R} N_{\theta \theta} \sin \beta=I_{0} \ddot{u}_{0} \\
&+I_{1} \ddot{\phi}_{x}, \\
& N_{x \theta, x}+\frac{1}{R} N_{\theta \theta, \theta}+\frac{2}{R} N_{x \theta} \sin \beta+\frac{1}{R} Q_{\theta} \cos \beta=I_{0} \ddot{v}_{0} \\
&+I_{1} \ddot{\phi}_{\theta}, \\
& Q_{x, x}+\frac{1}{R} Q_{\theta \theta, \theta}+\frac{1}{R} Q_{x} \sin \beta-\frac{1}{R} N_{\theta \theta} \cos \beta \\
&+N_{x x, x} \frac{\partial w_{0}}{\partial x}+N_{x x} \frac{\partial^{2} w_{0}}{\partial x^{2}}+\frac{1}{R} N_{x x} \frac{\partial w_{0}}{\partial x} \sin \beta \\
&+\frac{1}{R^{2}} N_{\theta \theta} \frac{\partial^{2} w_{0}}{\partial \theta^{2}}+\frac{1}{R^{2}} N_{\theta \theta, \theta} \frac{\partial w_{0}}{\partial \theta}+\frac{1}{R} N_{x \theta, \theta} \frac{\partial w_{0}}{\partial x} \\
&+\frac{2}{R} N_{x \theta} \frac{\partial^{2} w_{0}}{\partial x \partial \theta}+\frac{1}{R} N_{x \theta, x} \frac{\partial w_{0}}{\partial \theta}+N_{x x, x} \frac{\partial w^{*}}{\partial x} \\
&+N_{x x} \frac{\partial^{2} w^{*}}{\partial x^{2}}+\frac{1}{R^{2}} N_{\theta \theta} \frac{\partial^{2} w^{*}}{\partial \theta^{2}}+\frac{1}{R} N_{x x} \frac{\partial w^{*}}{\partial x} \sin \beta \\
&+\frac{1}{R^{2}} N_{\theta \theta, \theta} \frac{\partial w^{*}}{\partial \theta}+\frac{1}{R} N_{x \theta, x} \frac{\partial w^{*}}{\partial \theta}+\frac{1}{R} N_{x \theta, \theta} \frac{\partial w^{*}}{\partial x} \\
&+\frac{2}{R} N_{x \theta} \frac{\partial^{2} w^{*}}{\partial x \partial \theta}+F \cos \left(\Omega_{1} t\right)-\gamma \dot{w}_{0}=I_{0} \ddot{w}_{0}, \\
& M_{x x, x}+\frac{1}{R} M_{x \theta, \theta}-Q_{x}+\frac{1}{R} M_{x x} \sin \beta \\
&-\frac{1}{R} M_{\theta \theta} \sin \beta=I_{1} \ddot{u}_{0}+I_{2} \ddot{\phi}_{x}, \\
& M_{x \theta, x}+\frac{1}{R} M_{\theta \theta, \theta}-Q_{\theta}+\frac{2}{R} M_{x \theta} \sin \beta=I_{1} \ddot{v}_{0}+I_{2} \ddot{\phi}_{\theta},
\end{aligned}
$$

where $\mathbf{N}=\left[N_{x x}, N_{\theta \theta}, N_{x \theta}\right]^{\prime}$ and $\mathbf{Q}=\left[Q_{x}, Q_{\theta}\right]^{\prime}$ are the stress resultants and $\mathbf{M}=\left[M_{x x}, M_{\theta \theta}, M_{x \theta}\right]^{\prime}$ denotes the moment resultant; the primes represent the transposition of a matrix.

The stress resultants and the moment resultant can be rewritten by

$$
\begin{aligned}
\left\{\begin{array}{l}
\mathbf{N} \\
\mathbf{M}
\end{array}\right\} & =\left[\begin{array}{ll}
\mathbf{A} & \mathbf{B} \\
\mathbf{B} & \mathbf{D}
\end{array}\right]\left\{\begin{array}{l}
\left\{\varepsilon_{0}\right\} \\
\left\{\varepsilon_{1}\right\}
\end{array}\right\}+\left\{\begin{array}{l}
\mathbf{N}^{\mathbf{T}} \\
\mathbf{M}^{\mathbf{T}}
\end{array}\right\}, \\
\mathbf{Q} & =K \int_{-h / 2}^{h / 2}[\mathbf{A}]\left\{\begin{array}{l}
\gamma_{x z} \\
\gamma_{\theta z}
\end{array}\right\} d z,
\end{aligned}
$$

where $K$ is the shear correction factor, and the shear correction factor $K$ is chosen as $5 / 6$. Thermal stress and moment resultants are expressed as follows:

$$
\left[\begin{array}{cc}
N_{x x}^{T} & M_{x x}^{T} \\
N_{\theta \theta}^{T} & M_{\theta \theta}^{T} \\
N_{x \theta}^{T} & M_{x \theta}^{T}
\end{array}\right]=-\int_{-h / 2}^{h / 2}\left\{\begin{array}{c}
Q_{11}+Q_{12} \\
Q_{21}+Q_{22} \\
0
\end{array}\right\} \alpha(1, z) \Delta T d z .
$$

The extensional stiffness $A_{i j}$, the bending-extensional coupling stiffness $B_{i j}$, and the bending stiffness $D_{i j}$ of the imperfect FGM conical panel are calculated by

$$
\left(A_{i j}, B_{i j}, D_{i j}\right)=\int_{-h / 2}^{h / 2} Q_{i j}\left(1, z, z^{2}\right) d z .
$$

In addition, the mass inertia terms are defined as follows:

$$
\left(I_{0}, I_{1}, I_{2}\right)=\int_{-h / 2}^{h / 2}\left(1, z^{1}, z^{2}\right) \rho d z .
$$

Using the above equations, the nonlinear equations of motion in terms of generalized displacements can be obtained in the Appendix.

For the imperfect FGM conical panel, the simple supported boundary conditions are considered and expressed by

$$
\begin{array}{ll}
v_{0}=w_{0}=\phi_{\theta}=M_{x x}=0 & \text { at } x=0, x=L, \\
u_{0}=w_{0}=\phi_{x}=M_{\theta \theta}=0 & \text { at } \theta=0, \theta=\gamma .
\end{array}
$$

$u_{0}, v_{0}, w_{0}, \phi_{x}$, and $\phi_{\theta}$ of the FGM conical panel can be represented in the form of double Fourier series as follows:

$$
\begin{aligned}
& u_{0}=\sum_{e=1}^{E} \sum_{f=1}^{F} u_{e f}(t) \cos \left(\frac{m \pi x}{L}\right) \sin \left(\frac{n \pi \theta}{\gamma}\right), \\
& v_{0}=\sum_{e=1}^{E} \sum_{f=1}^{F} v_{e f}(t) \sin \left(\frac{m \pi x}{L}\right) \cos \left(\frac{n \pi \theta}{\gamma}\right), \\
& w_{0}=\sum_{e=1}^{E} \sum_{f=1}^{F} w_{e f}(t) \sin \left(\frac{m \pi x}{L}\right) \sin \left(\frac{n \pi \theta}{\gamma}\right), \\
& \phi_{x}=\sum_{e=1}^{E} \sum_{f=1}^{F} \phi_{x e f}(t) \cos \left(\frac{m \pi x}{L}\right) \sin \left(\frac{n \pi \theta}{\gamma}\right), \\
& \phi_{\theta}=\sum_{e=1}^{E} \sum_{f=1}^{F} \phi_{\theta e f}(t) \sin \left(\frac{m \pi x}{L}\right) \cos \left(\frac{n \pi \theta}{\gamma}\right),
\end{aligned}
$$

where $m$ is axial half waves number and $n$ is circumferential waves number. In this paper, the first two modes of transverse nonlinear oscillations are mainly considered.

According to studies given by Nosier and Reddy [31] and Bhimaraddi [32], nonlinear radial vibration of the imperfect FGM conical panel occupies main aspect of dynamical characteristics. It is reasonable that all the inertia terms in in-plane and rotations in nonlinear equations of motion given in the Appendix can be ignored since their influences 
TABLE 1: The temperature-dependent coefficients of SUS304 and $\mathrm{SI}_{3} \mathrm{~N}_{4}$.

\begin{tabular}{lcccccc}
\hline Coefficients & $E(\mathrm{~Pa})$ & $\begin{array}{c}\text { SUS304 } \\
\rho\left(\mathrm{kg} \cdot \mathrm{m}^{-3}\right)\end{array}$ & $\alpha\left(\mathrm{K}^{-1}\right)$ & $E(\mathrm{~Pa})$ & $\begin{array}{c}\mathrm{SI}_{3} \mathrm{~N}_{4} \\
\left(\mathrm{~kg} \cdot \mathrm{m}^{-3}\right)\end{array}$ \\
\hline$P_{-1}$ & 0 & 0 & 0 & 0 & 0 & 0 \\
$P_{0}$ & $201.04 \times 10^{9}$ & 8166 & $12.33 \times 10^{-6}$ & $348.43 \times 10^{9}$ & 2370 & $5.872 \times 10^{-6}$ \\
$P_{1}$ & $3.079 \times 10^{-4}$ & 0 & $8.08 \times 10^{-4}$ & $-3.07 \times 10^{-4}$ & 0 & $9.09 \times 10^{-4}$ \\
$P_{2}$ & $-6.53 \times 10^{-7}$ & 0 & 0 & $2.160 \times 10^{-7}$ & 0 & 0 \\
$P_{3}$ & 0 & 0 & 0 & $-8.95 \times 10^{-11}$ & 0 \\
\hline
\end{tabular}

are small compared to the radial inertia term. Substituting double Fourier series of (16) into (A.1), (A.2), (A.4), and (A.5), Galerkin integration procedure is utilized to acquire four constant coefficients coupled algebraic equations. Then, we can derive the displacements $u_{0}, v_{0}, \phi_{x}$, and $\phi_{\theta}$ with respect to the displacement $w_{0}$. Consequently, substituting the expressions about $u_{0}, v_{0}, \phi_{x}$, and $\phi_{\theta}$ into the ordinary differential equation obtained from (A.3), one can get nonlinear ordinary differential equations of this system in terms of radial displacements.

It is known that vibration of the first two modes for the system plays an important role during vibration. Amplitudes of the first two modes are the largest. Based on the results of convergence studies which have been done by the author and coworkers [33], it is reasonable for us to focus on radial nonlinear vibration of the imperfect FGM conical panel in the first two modes mainly

$$
\begin{aligned}
\ddot{w}_{1} & +\mu_{1} \dot{w}_{1}+\zeta_{11} w_{1}+\zeta_{12} w_{2}+\zeta_{13} w_{1}^{2}+\zeta_{14} w_{1} w_{2} \\
& +\zeta_{15} w_{2}^{2}+\zeta_{16} w_{1}^{3}+\zeta_{17} w_{1}^{2} w_{2}+\zeta_{18} w_{1} w_{2}^{2}+\zeta_{19} w_{2}^{3} \\
& +\zeta_{110}\left(p_{0}+p_{1} \cos \Omega_{2} t\right) w_{1}+c_{1}=\zeta_{111} F_{1} \cos \Omega_{1} t \\
\ddot{w}_{2} & +\mu_{2} \dot{w}_{2}+\zeta_{21} w_{1}+\zeta_{22} w_{2}+\zeta_{23} w_{1}^{2}+\zeta_{24} w_{1} w_{2} \\
& +\zeta_{25} w_{2}^{2}+\zeta_{26} w_{1}^{3}+\zeta_{27} w_{1}^{2} w_{2}+\zeta_{28} w_{1} w_{2}^{2}+\zeta_{29} w_{2}^{3} \\
& +\zeta_{210}\left(p_{0}+p_{1} \cos \Omega_{2} t\right) w_{2}+c_{2}=\zeta_{211} F_{2} \cos \Omega_{1} t
\end{aligned}
$$

where $\mu_{1}$ and $\mu_{2}$ represent the damping effects and $c_{1}$ and $c_{2}$ are the constants. The imperfection of FGM conical panel will change parameters, such as coefficients of the monomial and cubic stiffness terms, and parameters of constant terms.

\section{Numerical Results}

We choose the nonlinear ordinary differential equation (17) to conduct numerical simulations. A numerical approach through the computer software Matlab is utilized to explore complicated dynamic behaviors of the imperfect FGM conical panel. The Runge-Kutta algorithm and the Poincare map theory are used to construct numerical results of bifurcation diagrams. For the periodic motion, Poincare map consists of several separate points. For the chaotic motion, Poincare map consists of a number of points on the limited Poincare section. The periodic response and the chaotic response can be identified by several conventional criteria such as maximum
Lyapunov exponents, time histories, phase portraits, and Poincare maps.

The FGM conical panel is composed of two materials, which are the steel (SUS304) and the silicon nitride $\left(\mathrm{Si}_{3} \mathrm{~N}_{4}\right)$. The geometric parameters of the FGM conical panel are given as follows, where the slanted length is $L=2 \mathrm{~m}$, the thickness is $h=0.002 \mathrm{~m}$, the semivertex angle is $\beta=30^{\circ}$, the subtended angle is $\gamma=60^{\circ}$, and the radius at the top end is $R_{1}=0.5 \mathrm{~m}$. Table 1 shows the material properties of the FGM conical panel. The temperatures on the outer and inner surfaces are $T_{c}=400 \mathrm{~K}$ and $T_{m}=300 \mathrm{~K}$, respectively. The volume fraction index is chosen as $k=0.5$. The amplitudes of the radial excitation and the in-plane preapplied load are $F=5.0 \times 10^{5} \mathrm{~N} / \mathrm{m}^{2}$ and $p_{0}=6.0 \times 10^{6} \mathrm{~N} / \mathrm{m}^{2}$, respectively. The frequencies of the radial excitation and the in-plane excitation are selected as $\Omega_{1}=800 \mathrm{~Hz}$ and $\Omega_{2}=800 \mathrm{~Hz}$, respectively. The initial conditions in (17) are selected as $w_{1}=$ $-0.0010, \dot{w}_{1}=-0.0003, w_{2}=-0.00025$, and $\dot{w}_{2}=-0.0001$ in the following numerical simulation.

Since there are few literatures focused on the study of FGM conical panels in the thermal environment, especially taking into account the geometric imperfection, cylindrical panels are considered to validate the method. For this purpose, according to the Rayleigh-Ritz method, the dimensionless fundamental frequency $\bar{\omega}=\left(\omega L^{2} / h\right) \sqrt{\rho_{0 \mathrm{~m}} / E_{0 \mathrm{~m}}}$ is compared with the results from Shen and Wang [34] and Jooybar et al. [35] for the simply supported cylindrical panel with the geometric parameters as $h=0.001 \mathrm{~m}, L / R=1$, $L / h=20$, and $\gamma=180^{\circ} / \pi$, respectively. It is apparent from Table 2 that the present results are in good agreement with literatures and further verify the present approach.

3.1. Effect of Imperfection Types on Nonlinear Dynamics of the Structure. Based on (17), nonlinear dynamic analysis of the FGM conical panel with different imperfection types is investigated. Figure 3 illustrates the complicated dynamic behaviors of the conical panel without the imperfection when the in-plane excitation $p_{1}$ increases. It is found from Figure 3 that the motion of the system changes from the periodic motion to the chaotic motion. Figure 3(a) describes the bifurcation of transverse vibration for the first mode when the in-plane excitation increases from $2.50 \times 10^{7} \mathrm{~N} / \mathrm{m}^{2}$ to $3.50 \times 10^{7} \mathrm{~N} / \mathrm{m}^{2}$. Figure 3 (b) demonstrates the bifurcation of transverse vibration for the second mode when the parameters are selected the same as in Figure 3(a). Figure 3(c) is the three-dimensional bifurcation diagram of the first mode. Figure $3(\mathrm{~d})$ represents the maximum Lyapunov exponent 
TABLE 2: Comparisons of the natural frequencies $\bar{\omega}=\left(\omega L^{2} / h\right) \sqrt{\rho_{0 \mathrm{~m}} / E_{0 \mathrm{~m}}}$ of the FGM conical panel under the thermal environment $(h=$ $0.001 \mathrm{~m}, L / R=1, L / h=20$, and $\left.\gamma=180^{\circ} / \pi\right)$.

\begin{tabular}{|c|c|c|c|c|}
\hline \multirow{2}{*}{$k$} & & \multicolumn{3}{|c|}{ Temperature } \\
\hline & & $T_{c}=T_{m}=300 \mathrm{~K}$ & $T_{c}=T_{m}=400 \mathrm{~K}$ & $T_{c}=500 \mathrm{~K}, T_{m}=300 \mathrm{~K}$ \\
\hline \multirow{3}{*}{1} & Shen and Wang [34] & 15.9915 & 15.0425 & 15.1260 \\
\hline & Jooybar et al. [35] & 15.6320 & 15.4526 & 15.3647 \\
\hline & Present & 15.2476 & 15.1080 & 15.0054 \\
\hline \multirow{3}{*}{2} & Shen and Wang [34] & 14.2360 & 13,3045 & 13.4319 \\
\hline & Jooybar et al. [35] & 13.8891 & 13,7317 & 13.6868 \\
\hline & Present & 13.5172 & 13.4004 & 13.2811 \\
\hline
\end{tabular}

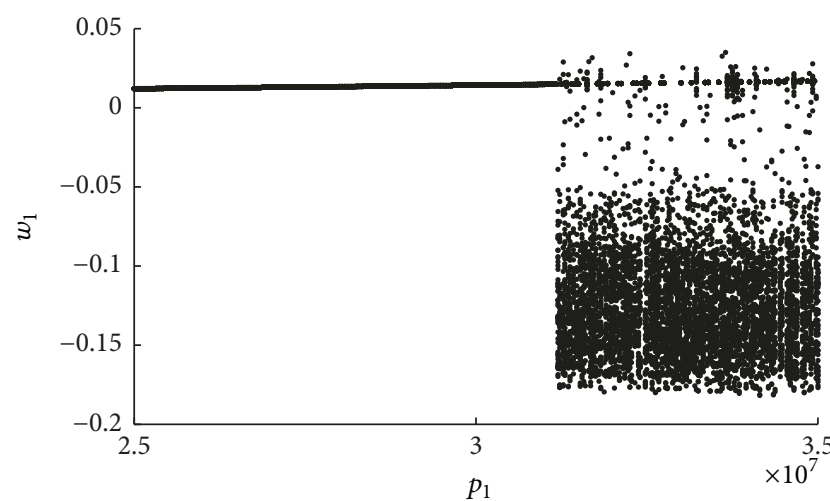

(a)

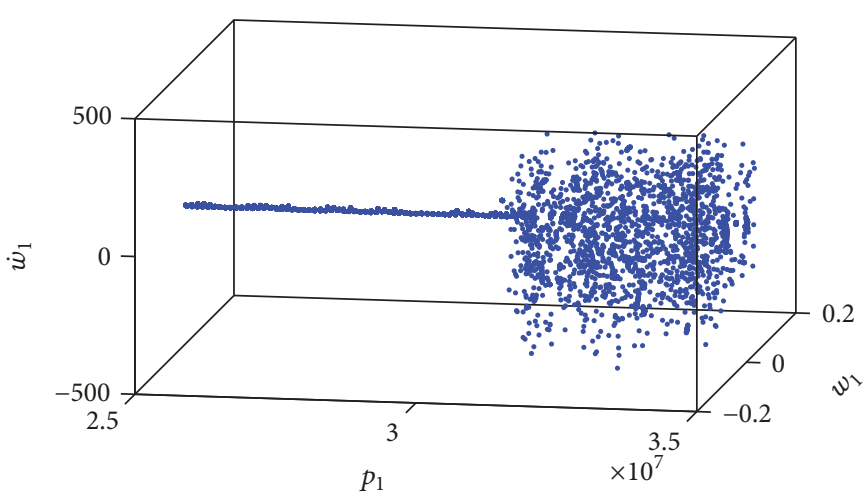

(c)

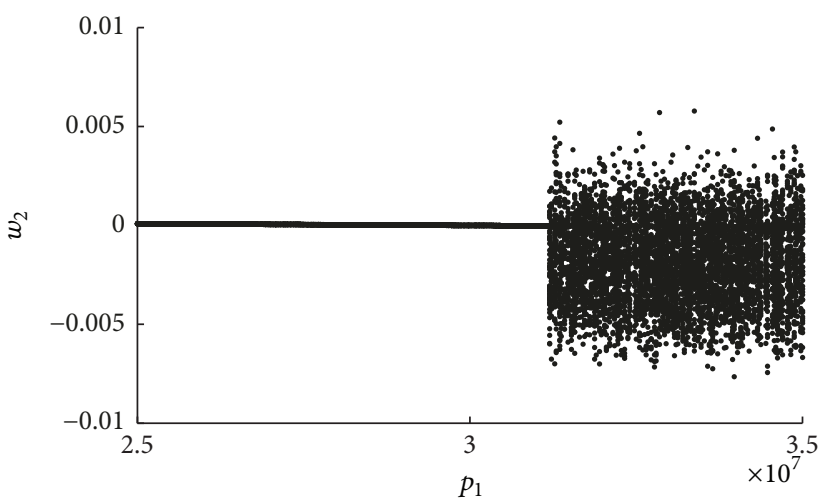

(b)

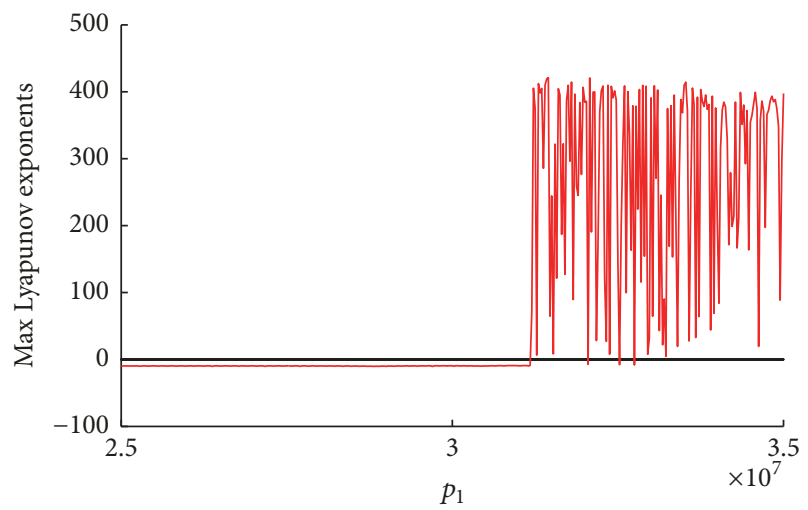

(d)

FIGURE 3: Bifurcation and maximum Lyapunov exponent of the FGM conical panel without imperfection with the in-plane excitation $p_{1}$ : (a) bifurcation diagram of the first mode; (b) bifurcation diagram of the second mode; (c) three-dimensional bifurcation diagram of the first mode; (d) maximum Lyapunov exponent diagram.

when parameters are chosen the same as the bifurcation diagrams. It is observed from Figure 3 that when the in-plane excitation is increased to $p_{1}=3.11 \times 10^{7} \mathrm{~N} / \mathrm{m}^{2}$, the motion of the FGM conical panel changes from the periodic motion to the chaotic motion. It can be known that the negative and positive Lyapunov exponents represent the periodic and chaotic motions, respectively.

Figure 4 shows the dynamic behavior of the FGM conical panel with the imperfection when the imperfection type is described by the form of $w^{*}=0.1 h \sin (\pi x / L) \sin (2 \pi \theta / \gamma)$. With the increase of the in-plane excitation, the motion law of the system is as follows: periodic motion $\rightarrow$ chaotic motion.
It can be seen from Figure 4 that the chaotic motion occurs in the system when the in-plane excitation is selected as $p_{1}=$ $3.10 \times 10^{7} \mathrm{~N} / \mathrm{m}^{2}$. Figure 5 illustrates the periodic motion of the FGM conical panel with the imperfection when the inplane excitation is chosen as $p_{1}=3.00 \times 10^{7} \mathrm{~N} / \mathrm{m}^{2}$, and other parameters are the same as in Figure 4. Figure 5(a) denotes the phase portrait on the plane $\left(w_{1}, \dot{w}_{1}\right)$ for the first mode. Figure 5(c) shows the phase portrait on the plane $\left(w_{2}, \dot{w}_{2}\right)$ for the second mode. Figures 5(b) and 5(d) are the time histories on the planes $\left(t, w_{1}\right)$ and $\left(t, w_{2}\right)$, respectively. Figure 5(e) represents the three-dimensional phase portrait in the space of $\left(w_{1}, \dot{w}_{1}, w_{2}\right)$. Figure 5(f) denotes Poincare map on the plane 


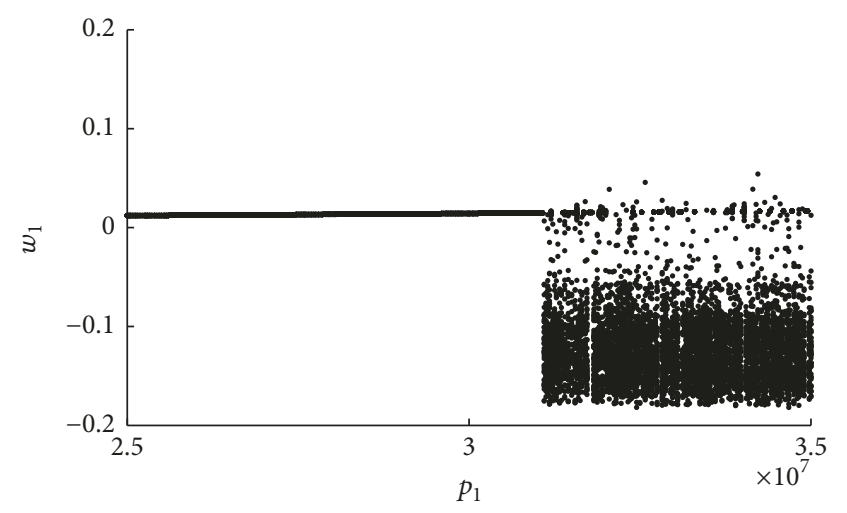

(a)

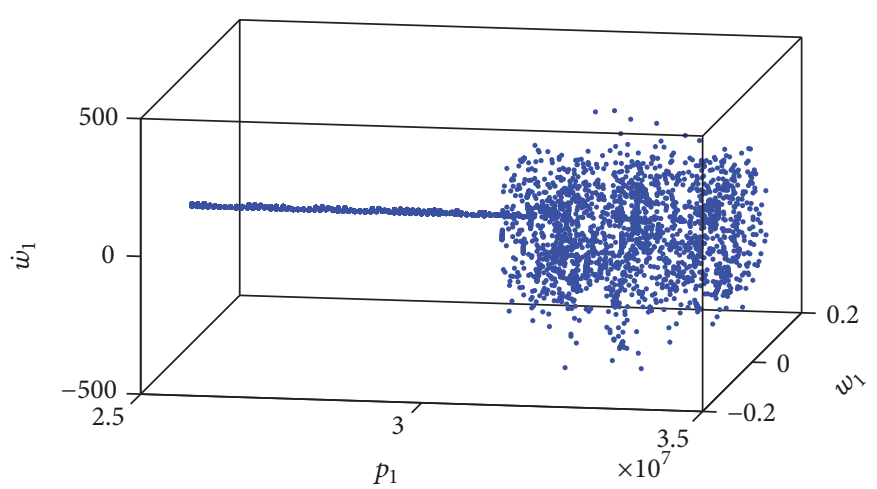

(c)

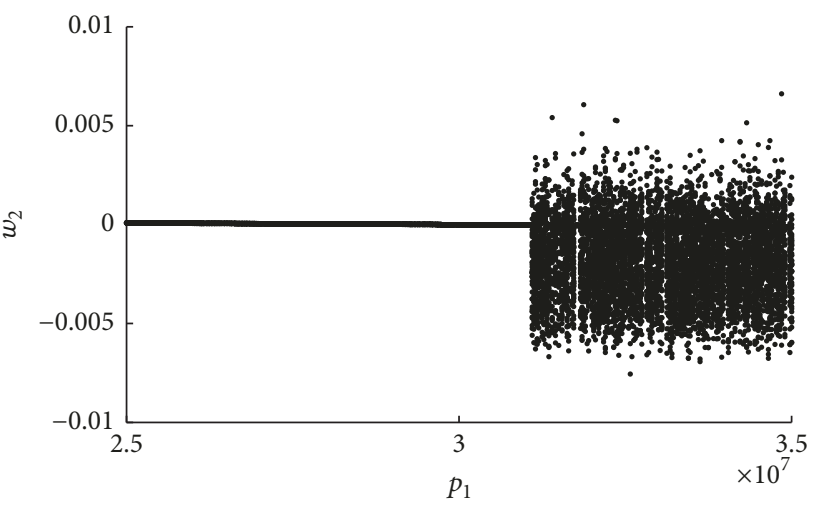

(b)

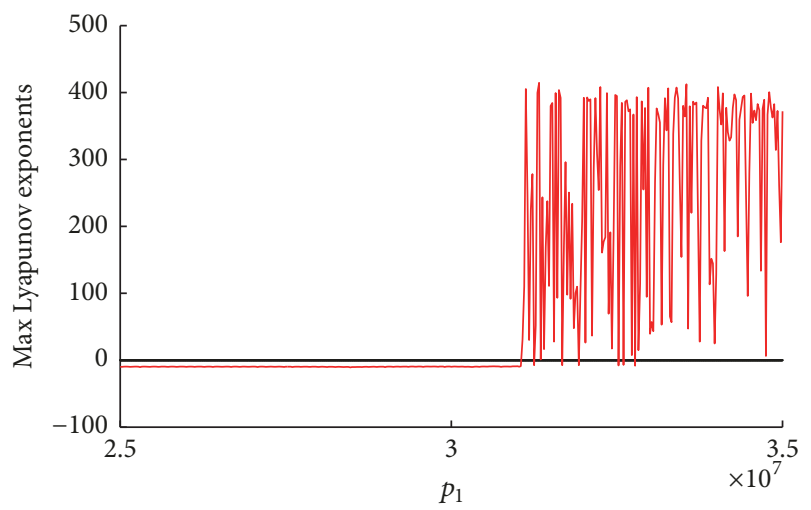

(d)

FIGURE 4: Bifurcation and maximum Lyapunov exponent diagrams with the in-plane excitation $p_{1}$, when the imperfection type of the FGM conical panel is $0.1 h \sin (\pi x / L) \sin (2 \pi \theta / \gamma)$ : (a) bifurcation diagram of the first mode; (b) bifurcation diagram of the second mode; (c) threedimensional bifurcation diagram of the first mode; (d) maximum Lyapunov exponent diagram.

$\left(w_{1}, \dot{w}_{1}\right)$ for the first mode. It can be seen from Figure 5(f) that Poincare map consists of only one point and the system appears to be in periodic motion. Figure 6 describes the chaotic motion of the system when the in-plane excitation is selected as $p_{1}=3.30 \times 10^{7} \mathrm{~N} / \mathrm{m}^{2}$, and other parameters are the same as in Figure 4. It can be observed from Figure 6(f) that Poincare map consists of a number of points on the limited Poincare section, and the system appears to be in chaotic motion.

Figure 7 shows the dynamic behavior of the FGM conical panel with the imperfection in the form of $w^{*}=$ $0.1 h \sin (\pi x / L)$ when half-wave number $\delta_{1}$ is chosen as $\delta_{1}=$ 1. It can be found from Figure 7 that the chaotic motion occurs in the system when the in-plane excitation is selected as $p_{1}=2.70 \times 10^{7} \mathrm{~N} / \mathrm{m}^{2}$.

Comparing with Figures 3, 4, and 7, it can be seen that the similar nonlinear dynamic behaviors occur in the system. But, the imperfection types of the FGM conical panel have an important effect on the periodic motion and the chaotic motion regions. When the imperfection type of the conical panel is chosen as $w^{*}=0.1 h \sin (\pi x / L)$, the chaotic motion is easier to appear in the system and the region of the chaotic motion is much wider, while the region of the chaotic motion for the FGM conical panel without the imperfection is much narrower. Based on the above analysis, it is found that the imperfection of the FGM conical panel influences characteristic of dynamics in the system.

\subsection{Effect of Half-Wave Numbers of Imperfection on Nonlinear} Dynamics of the Structure. Effects of half-wave numbers along the meridional direction on nonlinear vibration of the FGM conical panel are studied. When the imperfection type of the FGM conical panel is expressed by $0.1 h \sin \left(\delta_{1} \pi x / L\right)$ and half-wave number $\delta_{1}$ is taken as the value of 2 and 3, respectively, Figures 8 and 9 are obtained. Figure 8 demonstrates that the periodic motion begins to occur in the system when the in-plane excitation changes from $2.50 \times 10^{7} \mathrm{~N} / \mathrm{m}^{2}$ to $2.84 \times 10^{7} \mathrm{~N} / \mathrm{m}^{2}$ and half-wave number $\delta_{1}$ is selected as $\delta_{1}=2$. The chaotic motion appears in the region $p_{1} \in$ $\left(2.84 \times 10^{7} \mathrm{~N} / \mathrm{m}^{2}, 3.50 \times 10^{7} \mathrm{~N} / \mathrm{m}^{2}\right)$. Figure 9 depicts that the dynamic responses of the FGM conical panel present the law periodic motion $\rightarrow$ chaotic motion, when half-wave number $\delta_{1}$ is chosen as $\delta_{1}=3$. The periodic motion firstly appears in vibration of the FGM conical panel in the region $p_{1} \in\left(2.50 \times 10^{7} \mathrm{~N} / \mathrm{m}^{2}, 2.99 \times 10^{7} \mathrm{~N} / \mathrm{m}^{2}\right)$. The chaotic motion subsequently occurs in the FGM conical panel in the region $p_{1} \in\left(2.99 \times 10^{7} \mathrm{~N} / \mathrm{m}^{2}, 3.50 \times 10^{7} \mathrm{~N} / \mathrm{m}^{2}\right)$.

Comparing with Figures 7-9, it can be shown that the chaotic motion appears under the larger in-plane excitation 


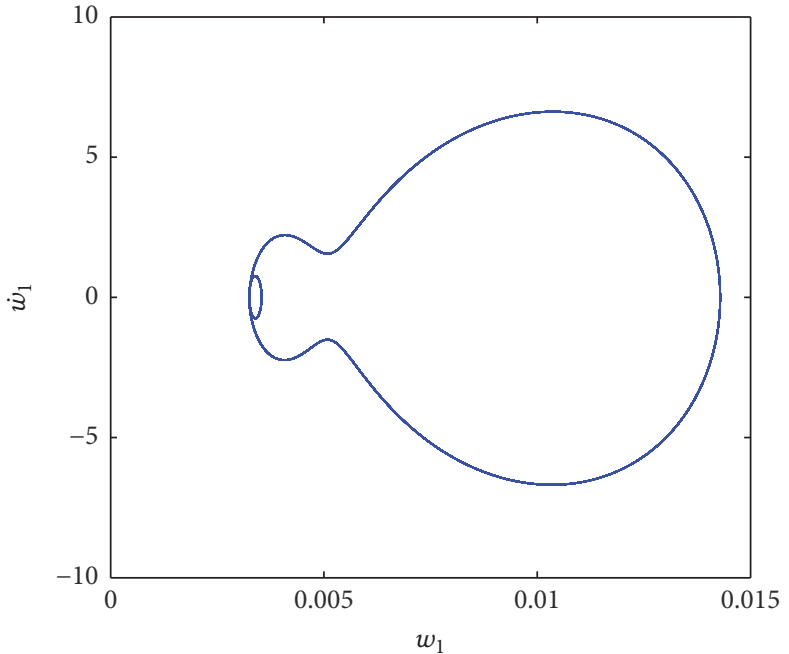

(a)

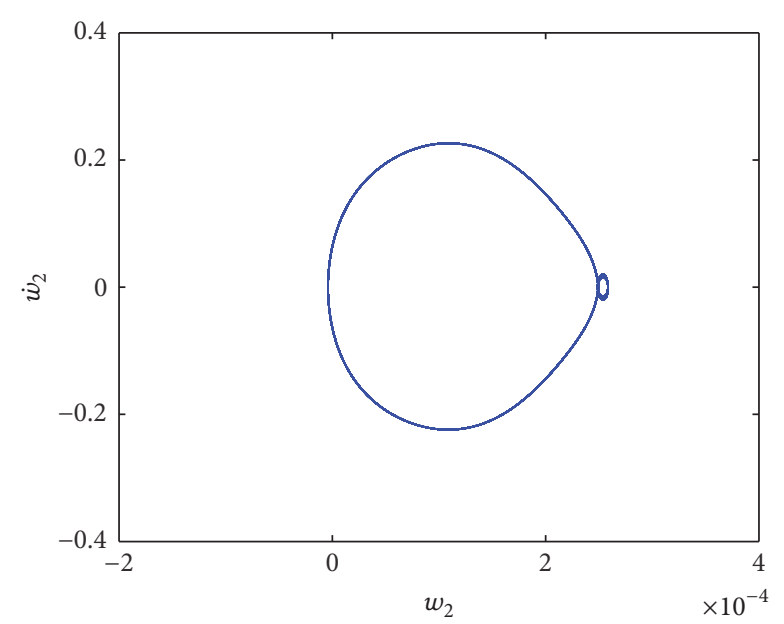

(c)

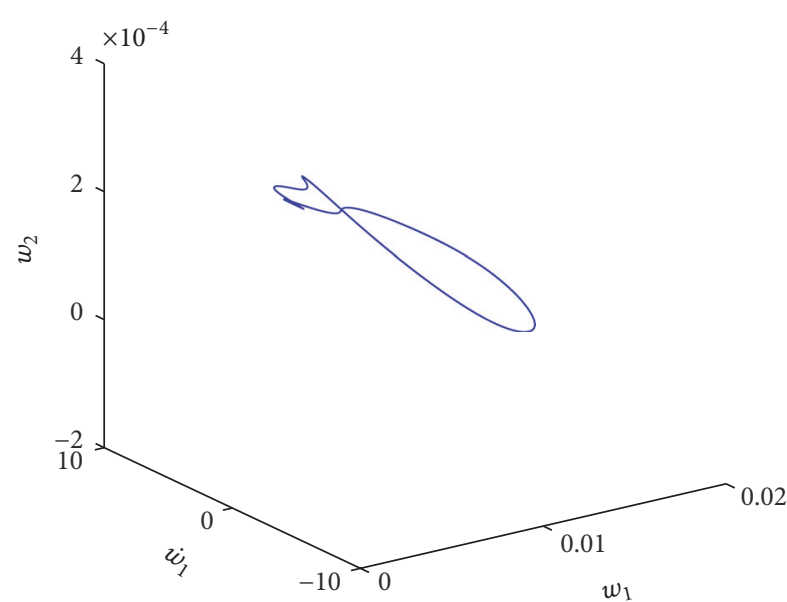

(e)

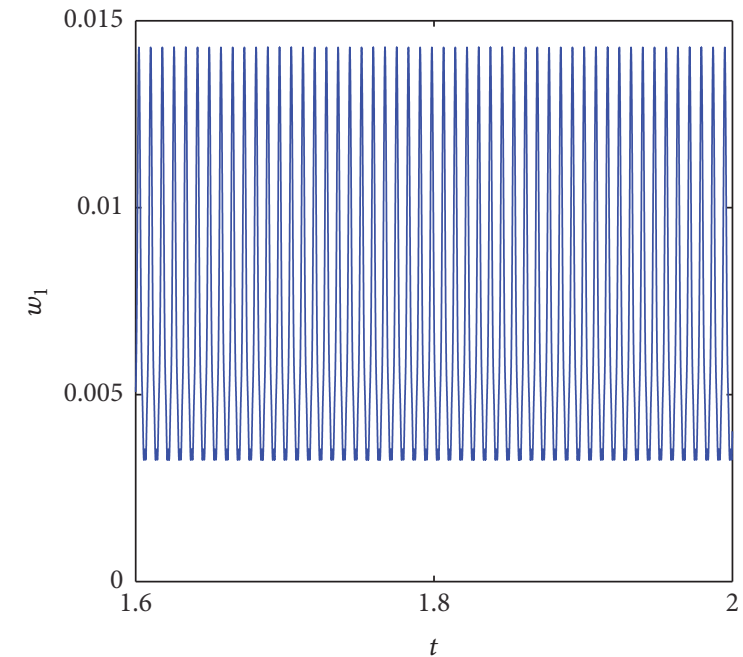

(b)

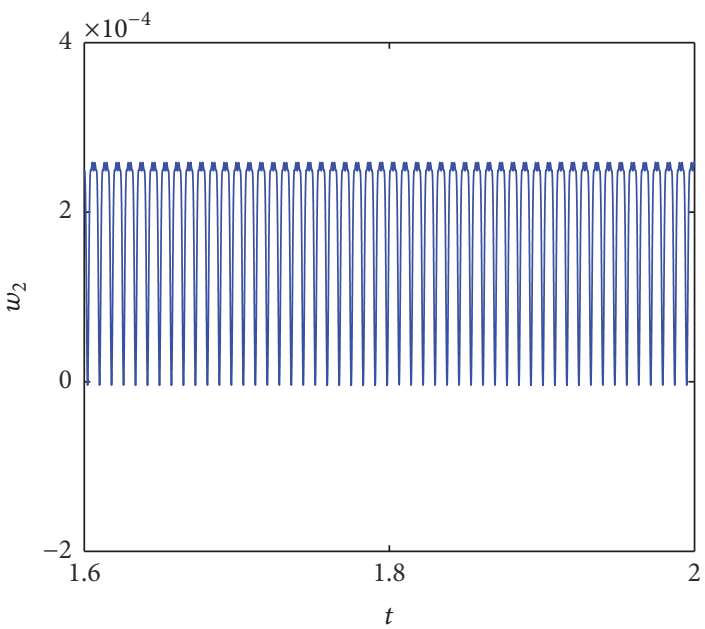

(d)

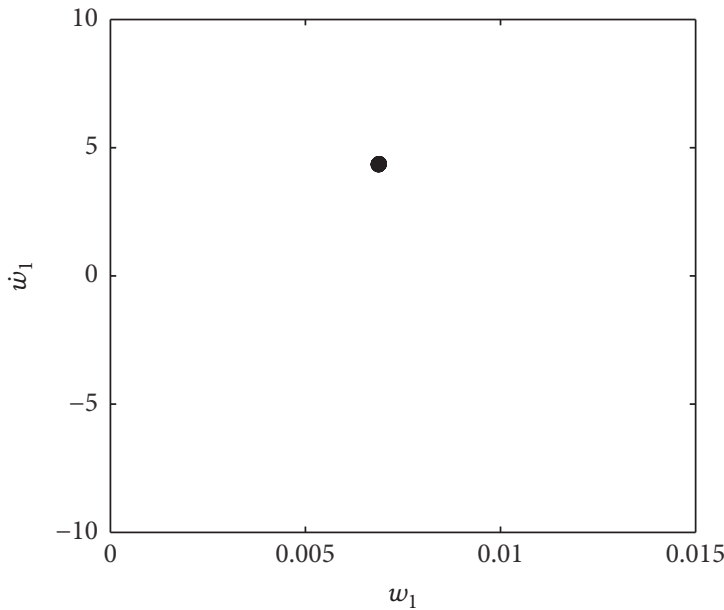

(f)

FIGURE 5: The periodic motion of the FGM conical panel with the imperfection of $0.1 h \sin (\pi x / L) \sin (2 \pi \theta / \gamma)$ occurs when the in-plane excitation is $p_{1}=3.0 \times 10^{7} \mathrm{~N} / \mathrm{m}^{2}$ : (a) the phase portrait on the plane $\left(w_{1}, \dot{w}_{1}\right)$; (b) the time history on the plane $\left(t, w_{1}\right)$; (c) the phase portrait on the plane $\left(w_{2}, \dot{w}_{2}\right)$; (d) the time history on the plane $\left(t, w_{2}\right)$; (e) the three-dimensional phase portrait in the space $\left(w_{1}, \dot{w}_{1}, w_{2}\right) ;(f)$ Poincare map on the plane $\left(w_{1}, \dot{w}_{1}\right)$. 


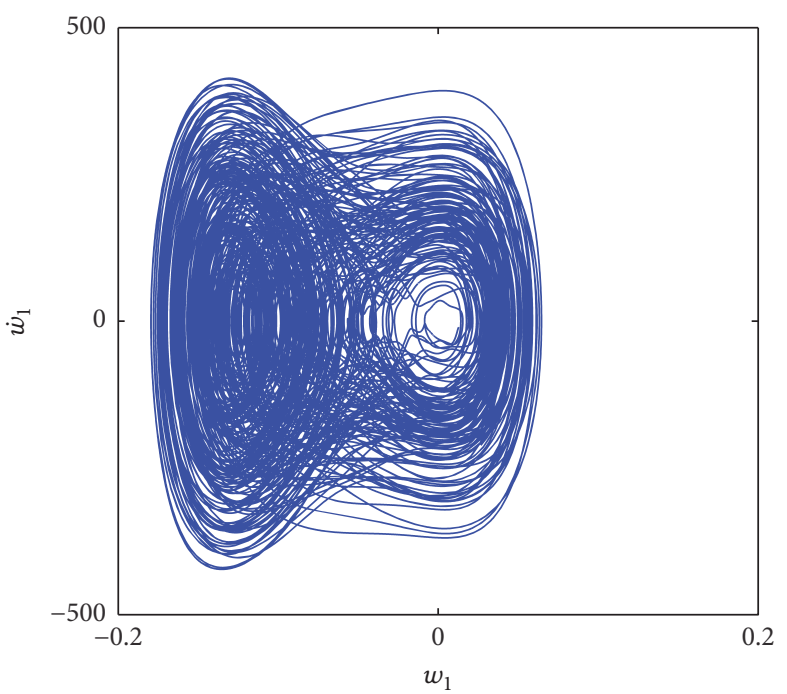

(a)

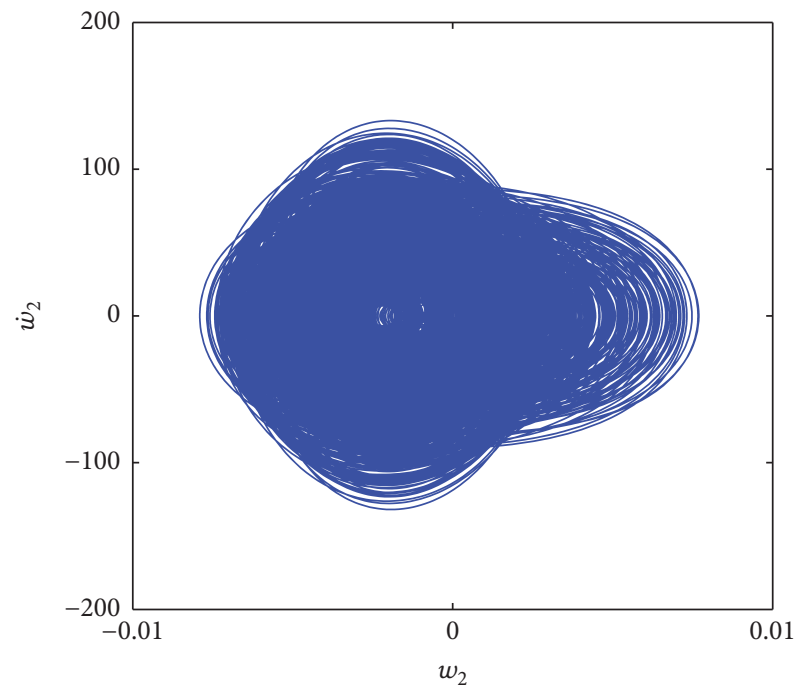

(c)

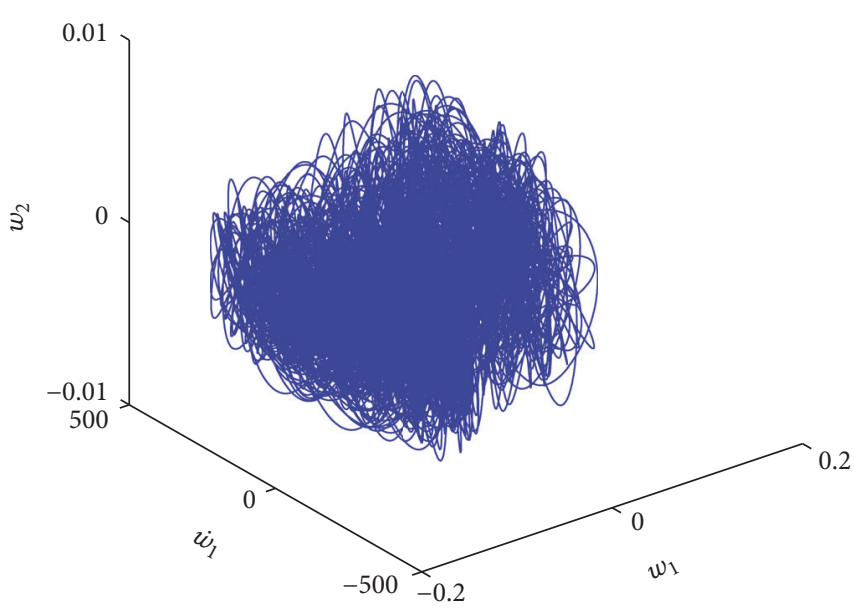

(e)

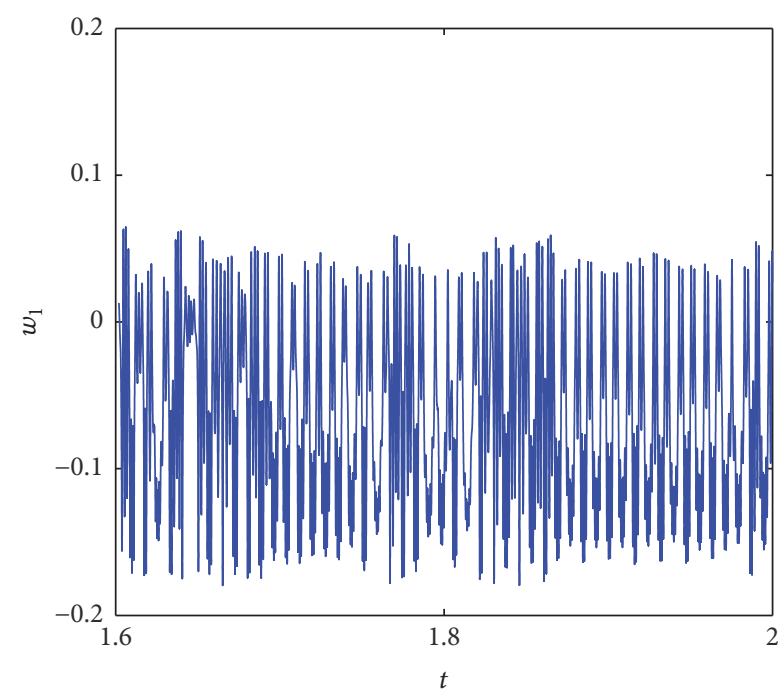

(b)

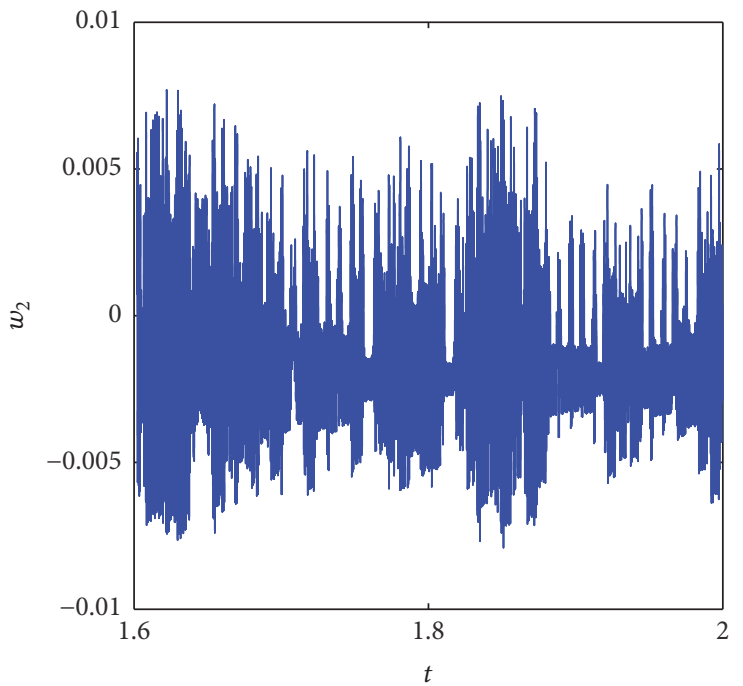

(d)

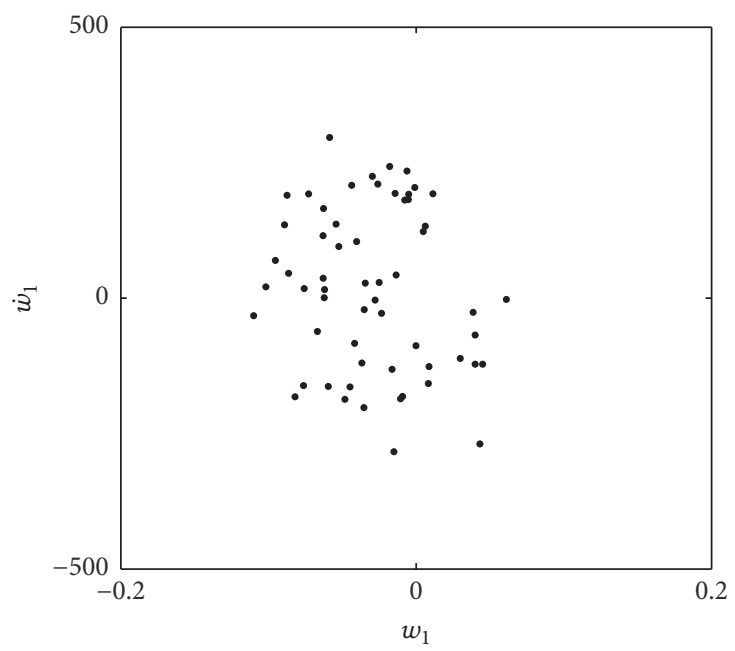

(f)

FIGURE 6: The chaotic motion of the FGM conical panel with the imperfection of $0.1 h \sin (\pi x / L) \sin (2 \pi \theta / \gamma)$ occurs when the in-plane excitation is $p_{1}=3.3 \times 10^{7} \mathrm{~N} / \mathrm{m}^{2}$ : (a) the phase portrait on the plane $\left(w_{1}, \dot{w}_{1}\right) ;(\mathrm{b})$ the time history on the plane $\left(t, w_{1}\right)$; (c) the phase portrait on the plane $\left(w_{2}, \dot{w}_{2}\right) ;(\mathrm{d})$ the time history on the plane $\left(t, w_{2}\right)$; (e) the three-dimensional phase portrait in the space $\left(w_{1}, \dot{w}_{1}, w_{2}\right) ;(\mathrm{f})$ Poincare map on the plane $\left(w_{1}, \dot{w}_{1}\right)$. 


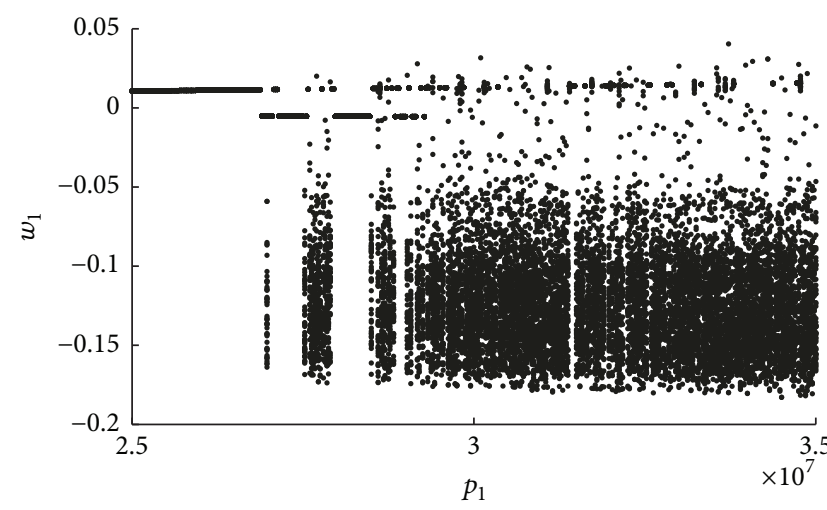

(a)

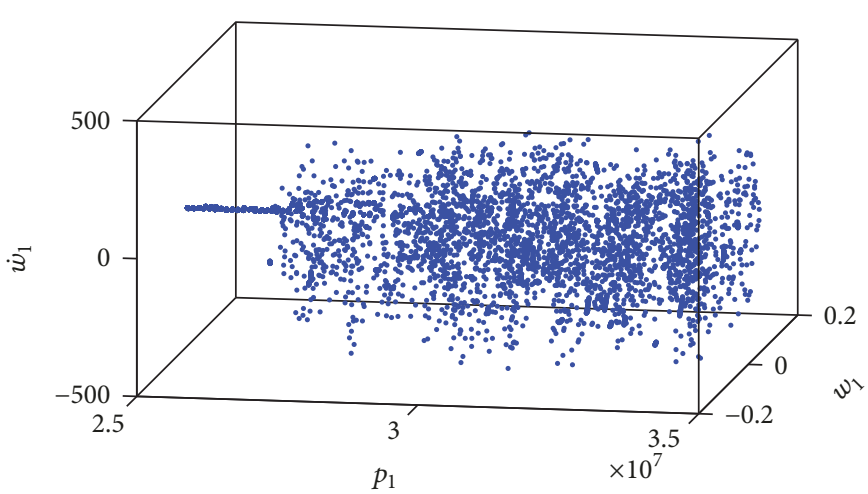

(c)

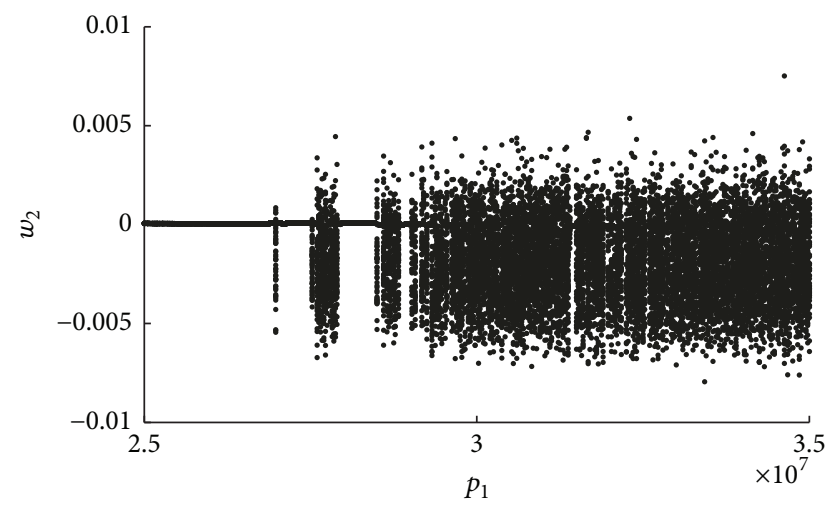

(b)

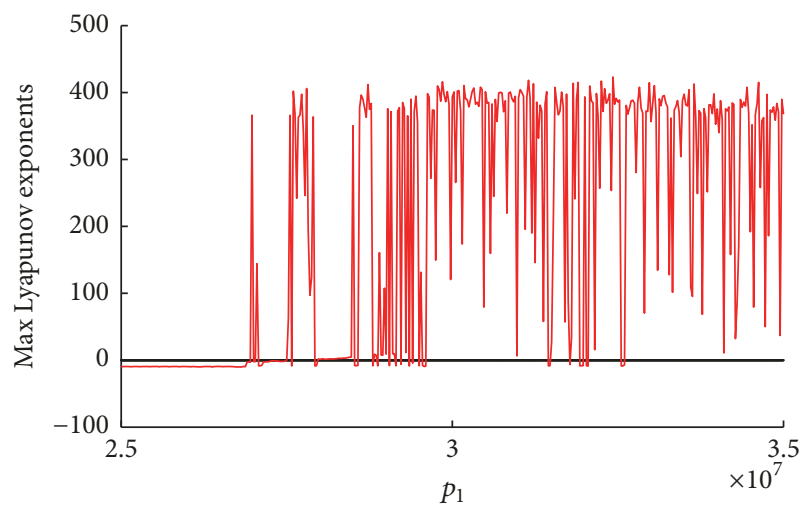

(d)

FIGURE 7: Bifurcation and maximum Lyapunov exponent diagrams with the in-plane excitation $p_{1}$, when the imperfection type of the FGM conical panel is $0.1 h \sin (\pi x / L)$ : (a) bifurcation diagram of the first mode; (b) bifurcation diagram of the second mode; (c) three-dimensional bifurcation diagram of the first mode; (d) maximum Lyapunov exponent diagram.

when the half-wave number of imperfection increases. This is the reason why the increases of half-wave number can improve the stiffness of the system.

\subsection{Effect of Amplitudes of Imperfection on Nonlinear Dynam-} ics of the Structure. Nonlinear dynamic analysis of the FGM conical panel with different amplitudes of the imperfection in the form of $w^{*}=\eta h \sin (3 \pi x / L)$ is discussed. Figure 10 shows the complicated nonlinear behaviors of the FGM conical panel when the amplitude of the imperfection $\eta$ is selected as 0.2. It is observed from Figure 10 that the chaotic motion appears in the system when the in-plane excitation is increased to $p_{1}=2.90 \times 10^{7} \mathrm{~N} / \mathrm{m}^{2}$, and other parameters are the same as in Figure 9. When the amplitude of the imperfection $\eta$ is chosen as 0.3 , bifurcation and maximum Lyapunov exponent diagrams are given, as shown in Figure 11. It is very obvious that the chaotic motion occurs in the system when the in-plane excitation becomes larger to be $p_{1}=$ $2.80 \times 10^{7} \mathrm{~N} / \mathrm{m}^{2}$. Comparing with Figures 9-11, it can be seen that the chaotic motion is easier to occur under the larger amplitude of the imperfection for the FGM conical panel. When the amplitude of the imperfection becomes smaller, characteristic of the dynamical behavior in the system usually presents the periodic motion. This is the reason that the more powerful the amplitude of the imperfection becomes, the greater the deformation that the FGM conical panel produces is, which makes the system cause the larger amplitude of vibration.

\subsection{Effect of Damping on Nonlinear Dynamics of the Structure.}

The influence of damping on the nonlinear dynamics of the FGM conical panel is depicted in Figure 12 when the imperfection in the form of $0.1 h \sin (3 \pi x / L)$ is given. In this case, the in-plane excitation is selected as $p_{1}=3.30 \times$ $10^{7} \mathrm{~N} / \mathrm{m}^{2}$, and other parameters are the same as in Figure 9 . It is obvious that, with the increase of the damping, dynamic responses of the system present the following evolution law: the system begins to enter into the region of the chaotic motion and then the periodic motion appears. It can be seen from Figure 12 that the periodic motion appears when the damping $\mu$ is increased to $\mu=100 \mathrm{~N} \cdot \mathrm{s} / \mathrm{m}$, where $\mu=\mu_{1}=\mu_{2}$. Based on the above analysis, it is found that the damping can significantly affect the nonlinear dynamic behaviors of the imperfect FGM conical panel.

\section{Conclusions}

Based on the first-order shear deformation theory and von Karman type nonlinear geometric relationship, the nonlinear 


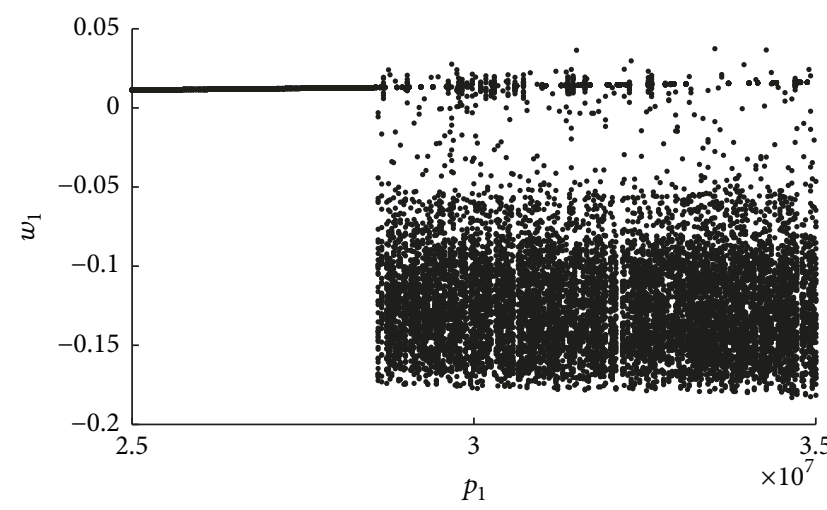

(a)

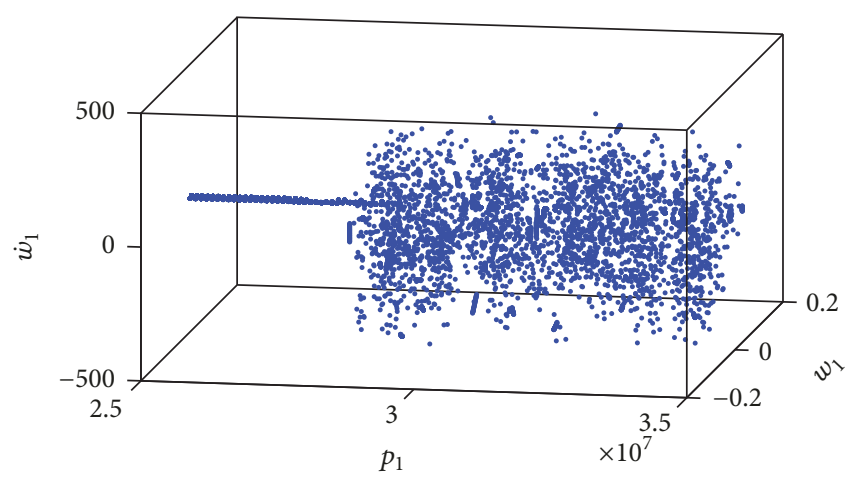

(c)

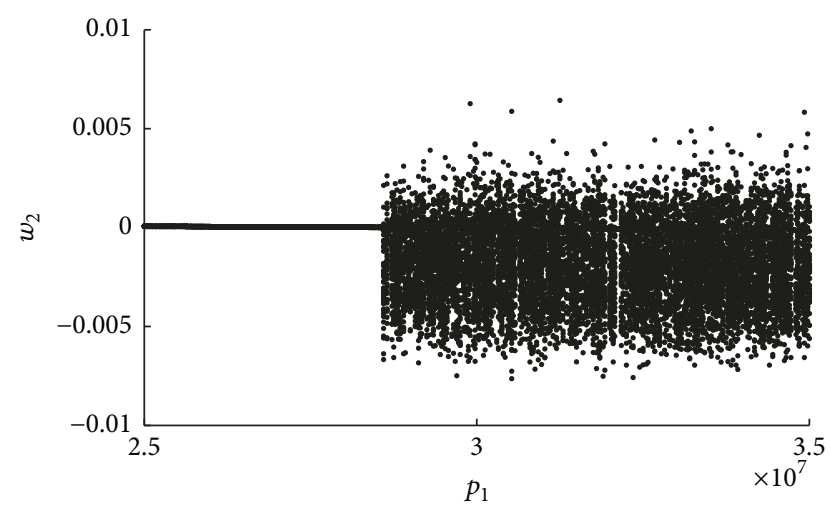

(b)

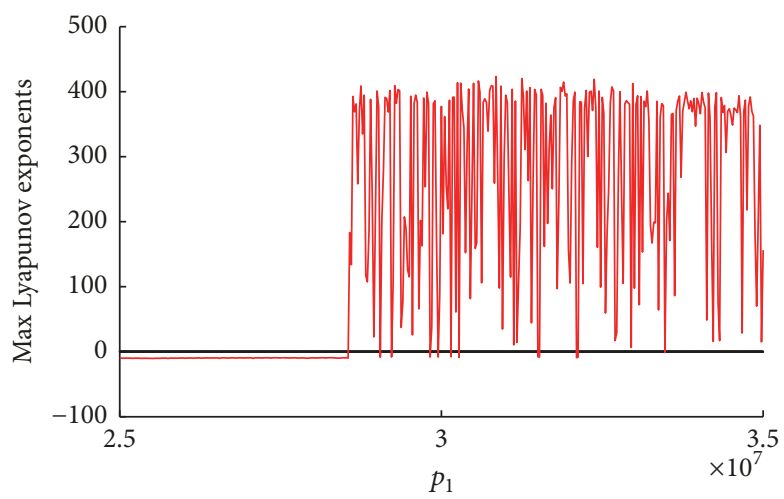

(d)

FIGURE 8: Bifurcation and maximum Lyapunov exponent diagrams with the in-plane excitation $p_{1}$, when the imperfection type of the FGM conical panel is $0.1 h \sin (2 \pi x / L)$ : (a) bifurcation diagram of the first mode; (b) bifurcation diagram of the second mode; (c) three-dimensional bifurcation diagram of the first mode; (d) maximum Lyapunov exponent diagram.

equations of motion for the FGM conical panel are established by using the Hamilton principle. The nonlinear partial differential governing equations are truncated by Galerkin method to obtain the ordinary differential equations along the radial displacement. The effects of imperfection types, half-wave numbers of the imperfection, amplitudes of the imperfection, and damping for the FGM conical panel are given.

Bifurcation diagrams, maximum Lyapunov exponents, phase portraits, time histories, and Poincare maps are presented to demonstrate the complicated nonlinear dynamic behaviors of the FGM conical panel. The following conclusions can be drawn:

(1) Compared with the FGM conical panel without the imperfection, the FGM conical panel with the imperfection is easier to show the chaotic motion and the region of the chaotic motion is much wider. The system is sensitive to the imperfection types, and the imperfection types of the FGM conical panel influence characteristic of dynamics in the system.

(2) With the increase of half-wave numbers of the imperfection, the chaotic motion appears under the larger in-plane excitation. This is the reason why the increase of half-wave numbers can improve the stiffness of the system.

(3) With the increase of amplitudes of the imperfection, the chaotic motion is easier to occur. This is the reason why the more powerful the amplitude of the imperfection becomes, the greater the deformation that the FGM conical panel produces is, which makes the system cause the larger amplitude of vibration.

(4) Analysis results show that the damping significantly affects the nonlinear dynamic behaviors of the imperfect FGM conical panel.

\section{Appendix}

Nonlinear equations of motion for the FGM conical panel with the imperfection are as follows:

$$
\begin{gathered}
A_{11} \frac{\partial^{2} u_{0}}{\partial x^{2}}+\frac{1}{R^{2}} A_{66} \frac{\partial^{2} u_{0}}{\partial \theta^{2}}+\frac{1}{R} A_{11} \frac{\partial u_{0}}{\partial x} \sin \beta \\
-\frac{1}{R^{2}} A_{22} u_{0} \sin ^{2} \beta+\frac{1}{R}\left(A_{12}+A_{66}\right) \frac{\partial^{2} v_{0}}{\partial x \partial \theta} \\
-\frac{1}{R^{2}}\left(A_{22}+A_{66}\right) \frac{\partial v_{0}}{\partial \theta} \sin \beta+\frac{1}{R^{2}} A_{66} \frac{\partial^{2} w_{0}}{\partial \theta^{2}} \frac{\partial w_{0}}{\partial x}
\end{gathered}
$$




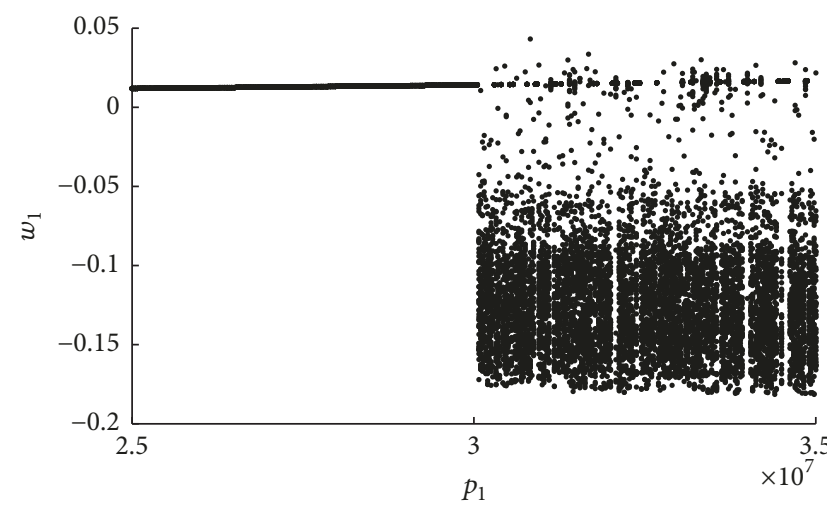

(a)

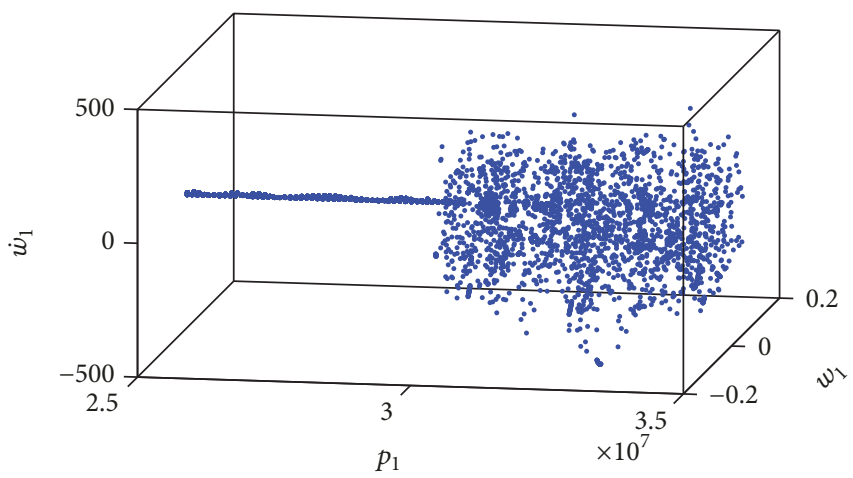

(c)

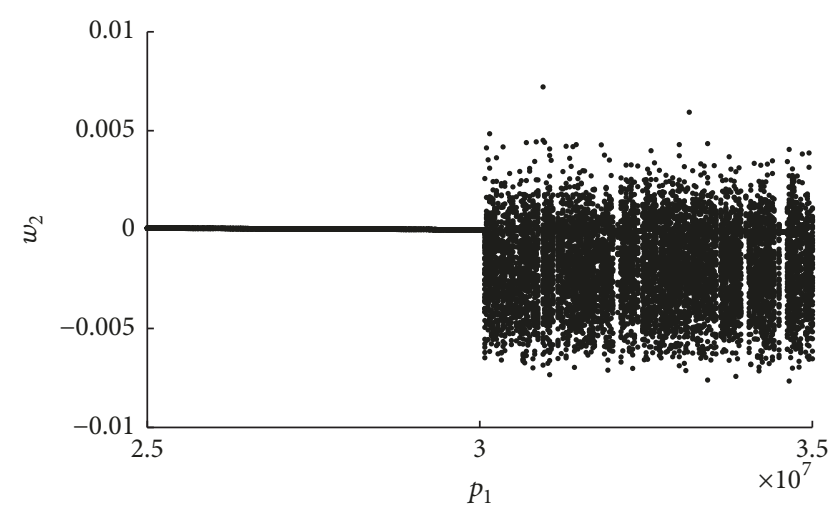

(b)

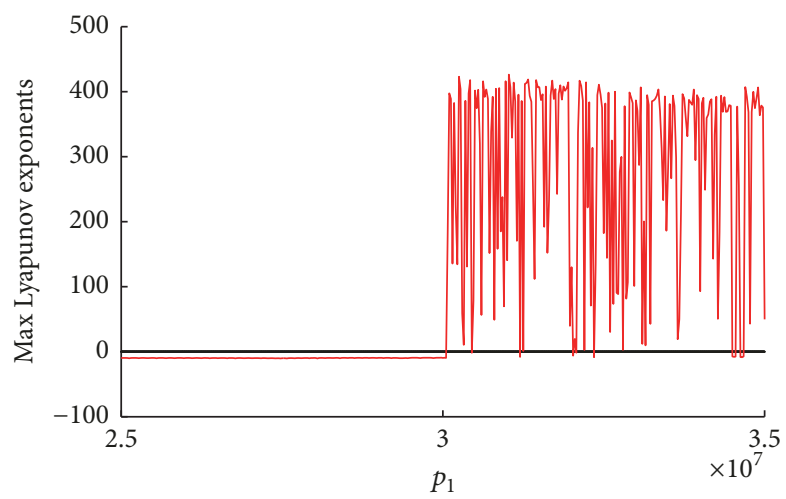

(d)

FIGURE 9: Bifurcation and maximum Lyapunov exponent diagrams with the in-plane excitation $p_{1}$, when the imperfection type of the FGM conical panel is $0.1 h \sin (3 \pi x / L)$ : (a) bifurcation diagram of the first mode; (b) bifurcation diagram of the second mode; (c) three-dimensional bifurcation diagram of the first mode; (d) maximum Lyapunov exponent diagram.

$$
\begin{aligned}
& +\frac{1}{R^{2}}\left(A_{12}+A_{66}\right) \frac{\partial^{2} w_{0}}{\partial x \partial \theta} \frac{\partial w_{0}}{\partial \theta}+B_{11} \frac{\partial^{2} \phi_{x}}{\partial x^{2}} \\
& +A_{11} \frac{\partial^{2} w_{0}}{\partial x^{2}} \frac{\partial w_{0}}{\partial x}+\frac{1}{R}\left(B_{12}+B_{66}\right) \frac{\partial^{2} \phi_{\theta}}{\partial x \partial \theta} \\
& +\frac{1}{2 R}\left(A_{11}-A_{12}\right) \frac{\partial^{2} w_{0}}{\partial x^{2}} \sin \beta+\frac{1}{R} A_{12} \frac{\partial w_{0}}{\partial x} \cos \beta \\
& -\frac{1}{2 R^{3}}\left(A_{12}+A_{22}\right) \frac{\partial^{2} w_{0}}{\partial \theta^{2}} \sin \beta \\
& -\frac{1}{R^{2}}\left(B_{22}+B_{66}\right) \frac{\partial \phi_{\theta}}{\partial \theta} \sin \beta+\frac{1}{R^{2}} B_{66} \frac{\partial^{2} \phi_{x}}{\partial \theta^{2}} \\
& +\frac{1}{R} B_{11} \frac{\partial \phi_{x}}{\partial x} \sin \beta-\frac{1}{R^{2}} A_{22} w_{0} \sin \beta \cos \beta \\
& -\frac{1}{R^{2}} B_{22} \phi_{x} \sin \beta+\frac{1}{R}\left(N_{x x}^{T}-N_{\theta \theta}^{T}\right) \sin \beta \\
& -\frac{1}{R^{2}} A_{22} w^{*} \sin \beta \cos \beta+\frac{1}{R} A_{12} \frac{\partial w^{*}}{\partial x} \cos \beta \\
& +\frac{1}{R^{2}} A_{66}\left(\frac{\partial^{2} w_{0}}{\partial x \partial \theta} \frac{\partial w^{*}}{\partial \theta}+\frac{\partial^{2} w_{0}}{\partial \theta^{2}} \frac{\partial w^{*}}{\partial x}\right)
\end{aligned}
$$

$$
\begin{aligned}
& +A_{11} \frac{\partial^{2} w_{0}}{\partial x^{2}} \frac{\partial w^{*}}{\partial x}+A_{11} \frac{\partial^{2} w^{*}}{\partial x^{2}} \frac{\partial w_{0}}{\partial x} \\
& +\frac{1}{R^{2}} A_{66} \frac{\partial^{2} w^{*}}{\partial \theta^{2}} \frac{\partial w_{0}}{\partial x}+\frac{1}{R^{2}} A_{12} \frac{\partial^{2} w^{*}}{\partial \theta^{2}} \frac{\partial w^{*}}{\partial x} \frac{\partial^{2} w_{0}}{\partial \theta^{2}} \\
& -\frac{2}{R^{3}} A_{12} \frac{\partial^{2} w^{*}}{\partial \theta^{2}} \frac{\partial^{2} w_{0}}{\partial \theta^{2}} \sin \beta+\frac{1}{R^{2}} A_{66} \frac{\partial^{2} w^{*}}{\partial x \partial \theta} \frac{\partial w_{0}}{\partial \theta} \\
& +\frac{1}{R}\left(A_{11}-A_{12}\right) \frac{\partial w^{*}}{\partial x} \frac{\partial w_{0}}{\partial x} \sin \beta \\
& +\frac{1}{R^{2}} A_{12} \frac{\partial^{2} w^{*}}{\partial \theta^{2}} \frac{\partial^{2} w_{0}}{\partial x \partial \theta} \frac{\partial w_{0}}{\partial \theta} \\
& +\frac{1}{R^{3}}\left(A_{12}-A_{22}\right) \frac{\partial w^{*}}{\partial \theta} \frac{\partial w_{0}}{\partial \theta} \sin \beta=I_{0} \ddot{u}_{0}+I_{1} \ddot{\phi}_{x} \\
& \frac{1}{R}\left(A_{12}+A_{66}\right) \frac{\partial^{2} u_{0}}{\partial x \partial \theta}+\frac{1}{R^{2}}\left(A_{22}+A_{66}\right) \frac{\partial u_{0}}{\partial \theta} \sin \beta \\
& +A_{66} \frac{\partial^{2} v_{0}}{\partial x^{2}}+\frac{1}{R^{2}} K A_{44} \frac{\partial w_{0}}{\partial \theta} \cos \beta+B_{66} \frac{\partial^{2} \phi_{\theta}}{\partial x^{2}} \\
& R^{2}
\end{aligned}
$$




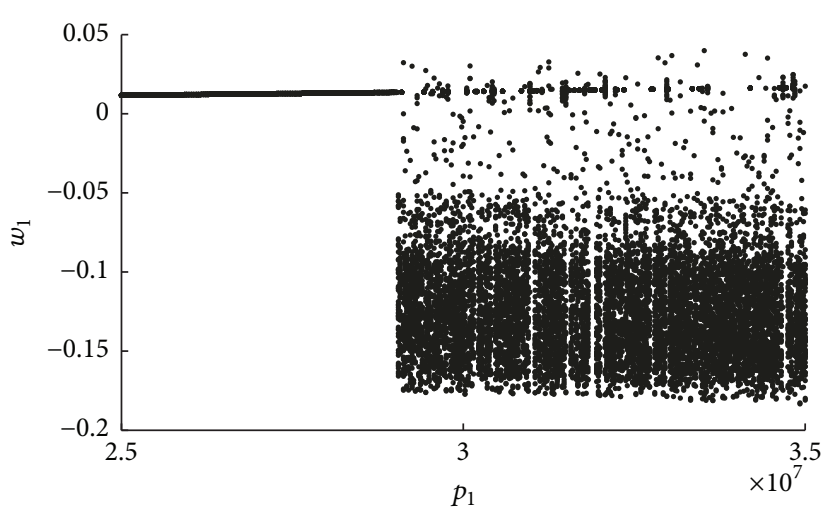

(a)

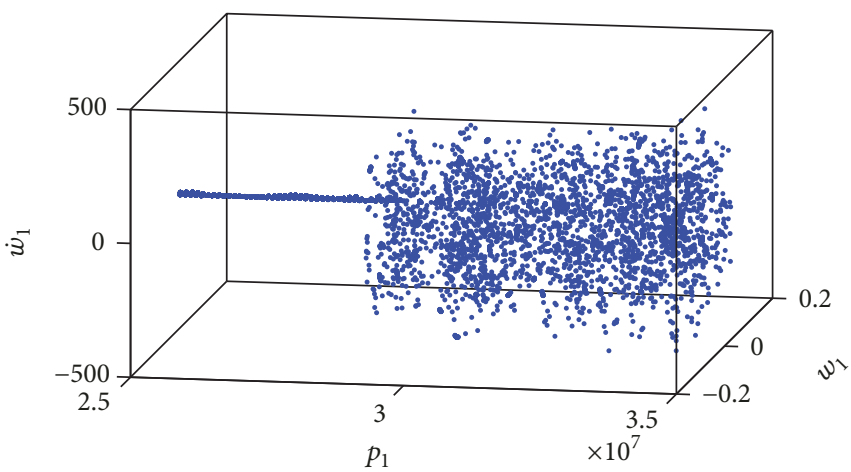

(c)

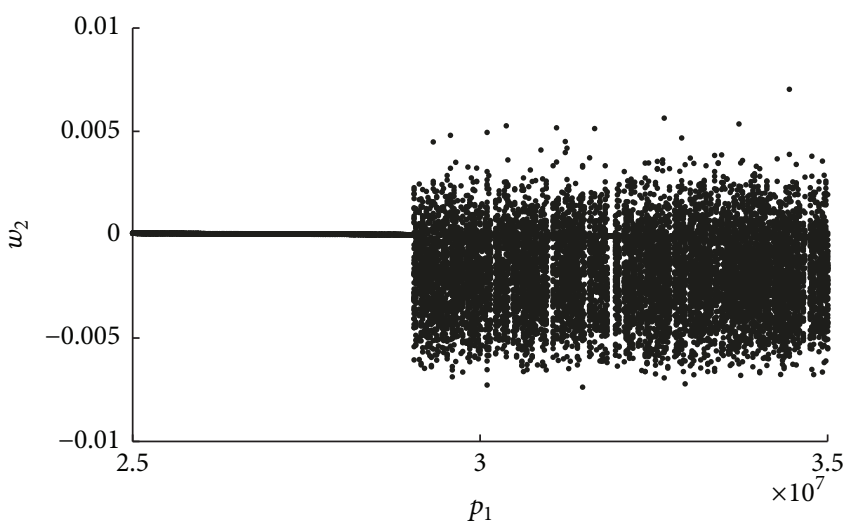

(b)

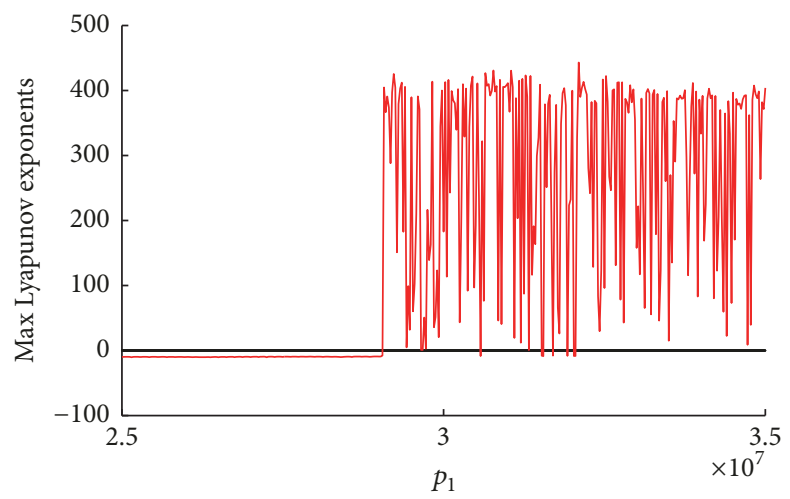

(d)

FIGURE 10: Bifurcation and maximum Lyapunov exponent diagrams with the in-plane excitation $p_{1}$, when the imperfection type of the FGM conical panel is $0.2 h \sin (3 \pi x / L)$ : (a) bifurcation diagram of the first mode; (b) bifurcation diagram of the second mode; (c) three-dimensional bifurcation diagram of the first mode; (d) maximum Lyapunov exponent diagram.

$$
\begin{aligned}
& +\frac{1}{R} A_{66} \frac{\partial w_{0}}{\partial \theta} \frac{\partial^{2} w_{0}}{\partial x^{2}}+\frac{1}{R^{3}} A_{22} \frac{\partial^{2} w_{0}}{\partial \theta^{2}} \frac{\partial w_{0}}{\partial \theta} \\
& +\frac{1}{R^{2}} B_{22} \frac{\partial^{2} \phi_{\theta}}{\partial \theta^{2}}+\frac{1}{R^{2}} A_{22} \frac{\partial^{2} v_{0}}{\partial \theta^{2}}+\frac{1}{R} A_{66} \frac{\partial v_{0}}{\partial x} \sin \beta \\
& +\frac{1}{R^{2}} A_{66} \frac{\partial w_{0}}{\partial \theta} \frac{\partial w_{0}}{\partial x} \sin \beta \\
& +\frac{1}{R^{2}}\left(B_{22}+B_{66}\right) \frac{\partial \phi_{x}}{\partial \theta} \sin \beta \\
& +\frac{1}{R}\left(A_{12}+A_{66}\right) \frac{\partial^{2} w_{0}}{\partial x \partial \theta} \frac{\partial w_{0}}{\partial x} \\
& +\frac{1}{R}\left(B_{12}+B_{66}\right) \frac{\partial^{2} \phi_{x}}{\partial x \partial \theta}+\frac{1}{R^{2}} A_{22} \frac{\partial w_{0}}{\partial \theta} \cos \beta \\
& +\frac{1}{R} B_{66} \frac{\partial \phi_{\theta}}{\partial x} \sin \beta \\
& +\left(\frac{1}{R} K A_{44} \cos \beta-\frac{1}{R^{2}} B_{66} \sin ^{2} \beta\right) \phi_{\theta} \\
& +\frac{1}{R^{2}} A_{66} \frac{\partial w^{*}}{\partial \theta} \frac{\partial w_{0}}{\partial x} \sin \beta+\frac{1}{R^{3}} A_{22} \frac{\partial^{2} w^{*}}{\partial \theta^{2}} \frac{\partial w_{0}}{\partial \theta}
\end{aligned}
$$$$
+\frac{1}{R}\left(A_{12}+A_{66}\right) \frac{\partial^{2} w^{*}}{\partial x \partial \theta} \frac{\partial w_{0}}{\partial x}+\frac{1}{R} A_{66} \frac{\partial^{2} w^{*}}{\partial x^{2}} \frac{\partial w_{0}}{\partial \theta}
$$$$
\begin{aligned}
& +\frac{1}{R^{2}} A_{66} \frac{\partial w^{*}}{\partial x} \frac{\partial w_{0}}{\partial \theta} \sin \beta+\frac{1}{R} A_{66} \frac{\partial^{2} w_{0}}{\partial x^{2}} \frac{\partial w^{*}}{\partial \theta} \\
& +\frac{1}{R}\left(A_{12}+A_{66}\right) \frac{\partial w^{*}}{\partial x} \frac{\partial^{2} w_{0}}{\partial x \partial \theta} \\
& +\frac{1}{R^{2}}\left(A_{22}+K A_{44}\right) \frac{\partial w^{*}}{\partial \theta} \cos \beta \\
& +\frac{1}{R^{3}} A_{22} \frac{\partial w^{*}}{\partial \theta} \frac{\partial^{2} w_{0}}{\partial \theta^{2}}=I_{0} \ddot{v}_{0}+I_{1} \ddot{\phi}_{\theta}
\end{aligned}
$$$$
\frac{2}{R^{2}} B_{66} \frac{\partial^{2} w_{0}}{\partial x \partial \theta} \frac{\partial \phi_{x}}{\partial \theta}+\frac{1}{2 R} A_{11}\left(\frac{\partial w_{0}}{\partial x}\right)^{3} \sin \beta
$$$$
-\frac{1}{R^{2}} A_{66} \frac{\partial v_{0}}{\partial \theta} \frac{\partial w_{0}}{\partial x} \sin \beta
$$$$
+\frac{1}{R}\left(B_{11}+B_{22}\right) \frac{\partial \phi_{x}}{\partial x} \frac{\partial w_{0}}{\partial x}-\frac{1}{R} N_{\theta \theta}^{T} \cos \beta
$$$$
+A_{11} \frac{\partial^{2} u_{0}}{\partial x^{2}} \frac{\partial w_{0}}{\partial x}-\frac{1}{R^{3}}\left(A_{12}+A_{66}\right) \frac{\partial^{2} w_{0}}{\partial \theta^{2}} \frac{\partial w_{0}}{\partial x}
$$ 


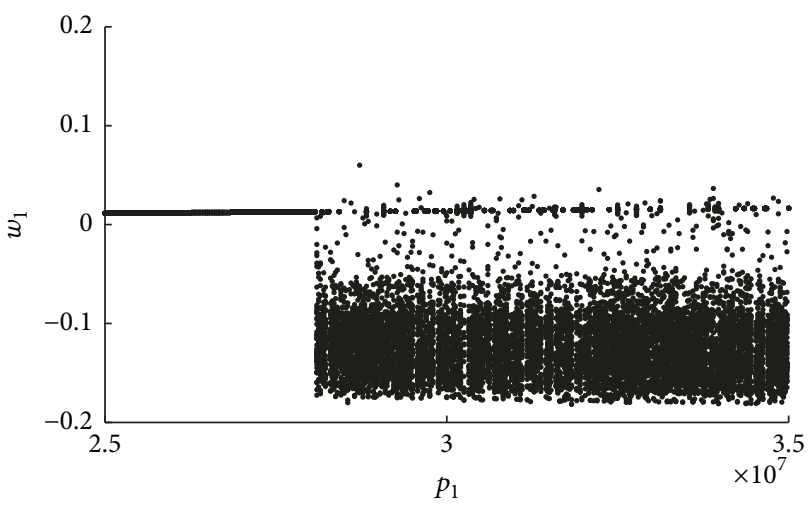

(a)

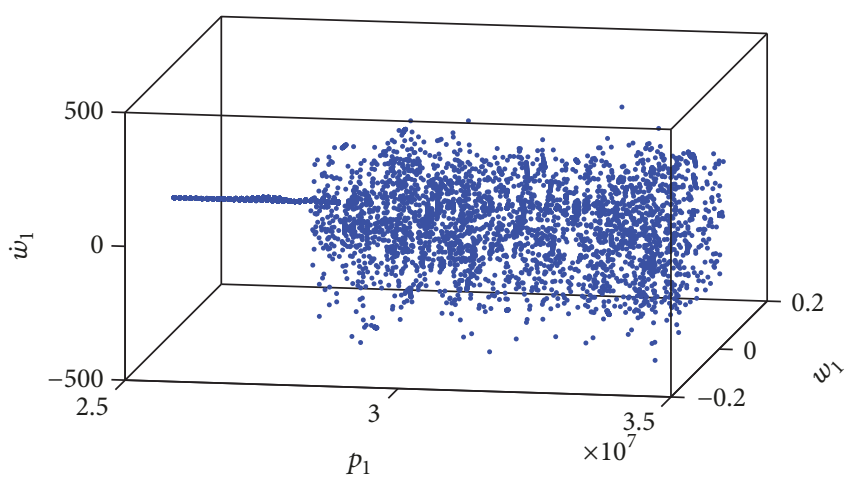

(c)

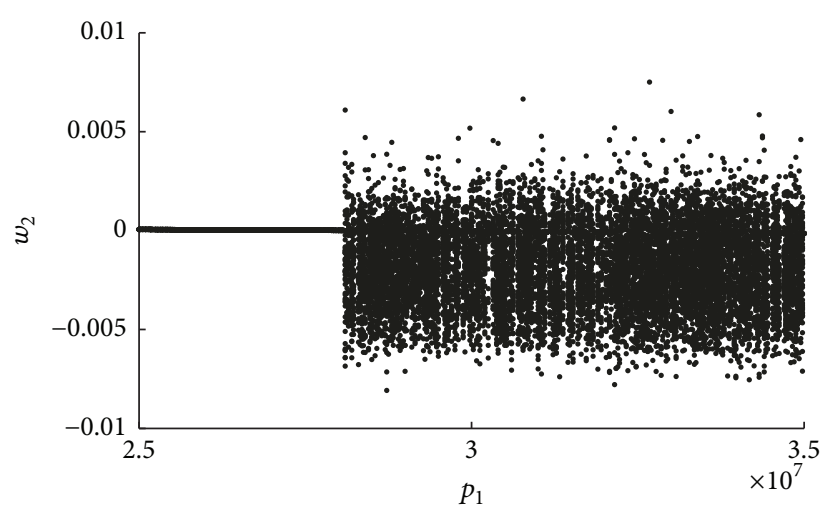

(b)

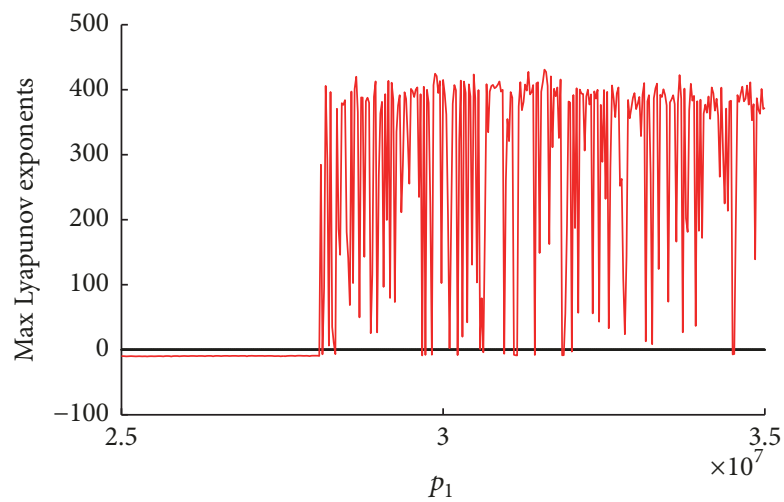

(d)

FIGURE 11: Bifurcation and maximum Lyapunov exponent diagrams with the in-plane excitation $p_{1}$, when the imperfection type of the FGM conical panel is $0.3 h \sin (3 \pi x / L)$ : (a) bifurcation diagram of the first mode; (b) bifurcation diagram of the second mode; (c) three-dimensional bifurcation diagram of the first mode; (d) maximum Lyapunov exponent diagram.

$$
\begin{aligned}
& +\frac{1}{R}\left(B_{12}+B_{66}\right) \frac{\partial^{2} \phi_{\theta}}{\partial x \partial \theta} \frac{\partial w_{0}}{\partial x} \\
& +\frac{2}{R^{2}}\left(A_{12}+2 A_{66}\right) \frac{\partial^{2} w_{0}}{\partial x \partial \theta} \frac{\partial w_{0}}{\partial \theta} \frac{\partial w_{0}}{\partial x} \\
& +B_{11} \frac{\partial^{2} \phi_{x}}{\partial x^{2}} \frac{\partial w_{0}}{\partial x}+\frac{1}{R}\left(K A_{55}+N_{x x}^{T}\right) \frac{\partial w_{0}}{\partial x} \sin \beta \\
& -\frac{1}{R^{2}} B_{66} \frac{\partial \phi_{\theta}}{\partial x} \frac{\partial w_{0}}{\partial x} \sin \beta+\frac{3}{2} A_{11} \frac{\partial^{2} w_{0}}{\partial x^{2}}\left(\frac{\partial w_{0}}{\partial x}\right)^{2} \\
& +\frac{1}{R^{2}} A_{66} \frac{\partial^{2} u_{0}}{\partial \theta^{2}} \frac{\partial w_{0}}{\partial x}+\frac{1}{2 R} A_{12}\left(\frac{\partial w_{0}}{\partial x}\right)^{2} \cos \beta \\
& +\frac{1}{2 R^{2}}\left(A_{12}+2 A_{66}\right) \frac{\partial^{2} w_{0}}{\partial \theta^{2}}\left(\frac{\partial w_{0}}{\partial x}\right)^{2} \\
& +\frac{1}{R}\left(A_{11}+A_{12}\right) \frac{\partial w_{0}}{\partial x} \frac{\partial u_{0}}{\partial x} \sin \beta \\
& +\frac{1}{R} B_{12} \frac{\partial^{2} w_{0}}{\partial x^{2}} \phi_{x} \sin \beta+\frac{1}{R} A_{12} \frac{\partial^{2} w_{0}}{\partial x^{2}} w_{0} \cos \beta \\
& +\frac{1}{R^{2}} A_{12} \frac{\partial^{2} w_{0}}{\partial \theta^{2}} \frac{\partial u_{0}}{\partial x}+A_{11} \frac{\partial^{2} w_{0}}{\partial x^{2}} \frac{\partial u_{0}}{\partial x}
\end{aligned}
$$

$$
\begin{aligned}
& +\frac{1}{R^{3}} A_{22} \frac{\partial^{2} w_{0}}{\partial \theta^{2}} w_{0} \cos \beta+\frac{2}{R} A_{66} \frac{\partial^{2} w_{0}}{\partial x \partial \theta} \frac{\partial v_{0}}{\partial x} \\
& -\frac{1}{R^{2}} A_{66} \frac{\partial w_{0}}{\partial \theta} \frac{\partial v_{0}}{\partial x}-A_{12} \frac{\partial u_{0}}{\partial x} \cos \beta \\
& -\frac{1}{R^{2}} A_{22} w_{0} \cos \beta+\frac{1}{R^{3}} B_{22} \frac{\partial^{2} w_{0}}{\partial \theta^{2}} \phi_{x} \sin \beta \\
& +\frac{1}{R}\left(A_{12}+A_{66}\right) \frac{\partial^{2} v_{0}}{\partial x \partial \theta} \frac{\partial w_{0}}{\partial x} \\
& +\left(\frac{1}{R} K A_{55}-\frac{1}{R^{2}} B_{22} \cos \beta\right) \phi_{x} \sin \beta \\
& +\frac{1}{R^{3}} B_{66} \frac{\partial w_{0}}{\partial \theta} \phi_{\theta} \sin ^{2} \beta-\frac{2}{R^{2}} B_{66} \frac{\partial^{2} w_{0}}{\partial x \partial \theta} \phi_{\theta} \sin \beta \\
& +\frac{1}{R^{3}} A_{22} \frac{\partial^{2} w_{0}}{\partial \theta^{2}} u_{0} \sin \beta-\frac{1}{R^{2}} A_{22} u_{0} \sin \beta \cos \beta \\
& +\frac{1}{R^{3}} A_{66} \frac{\partial^{2} v_{0}}{\partial \theta^{2}}+\frac{1}{R^{3}}\left(B_{22}-B_{66}\right) \frac{\partial \phi_{x}}{\partial \theta} \frac{\partial w_{0}}{\partial \theta} \\
& +\left(K A_{55}+N_{x x}^{T}-\left(p_{0}+p_{1} \cos \Omega_{2} t\right)\right) \frac{\partial^{2} w_{0}}{\partial x^{2}}
\end{aligned}
$$




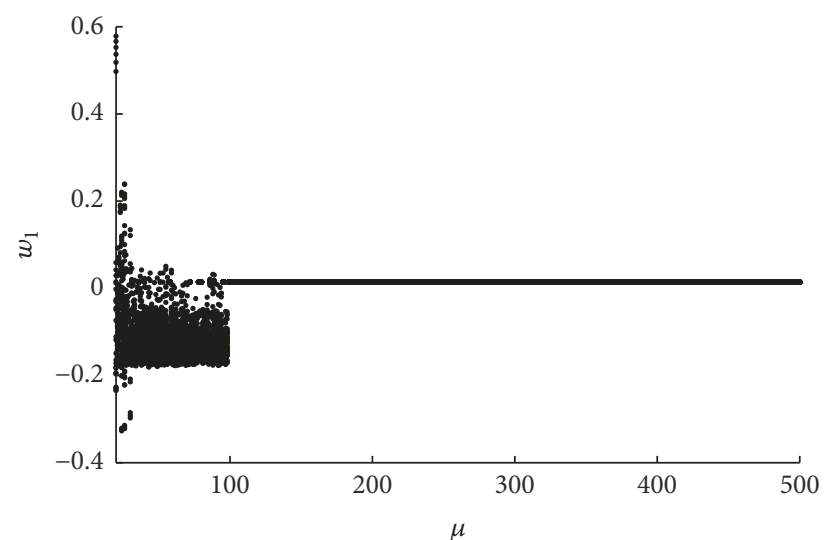

(a)

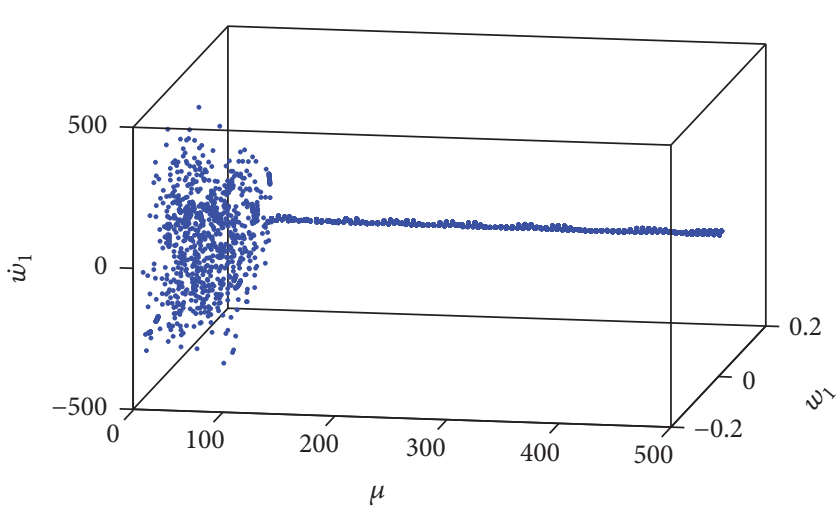

(c)

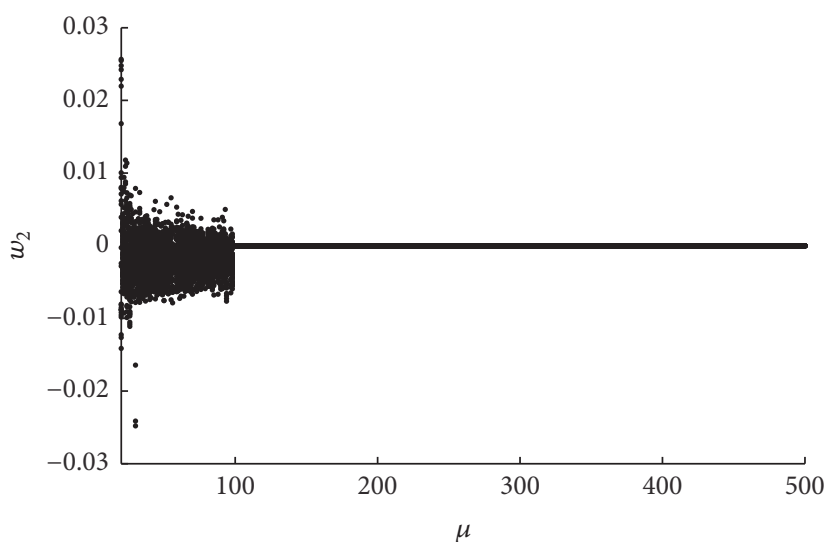

(b)

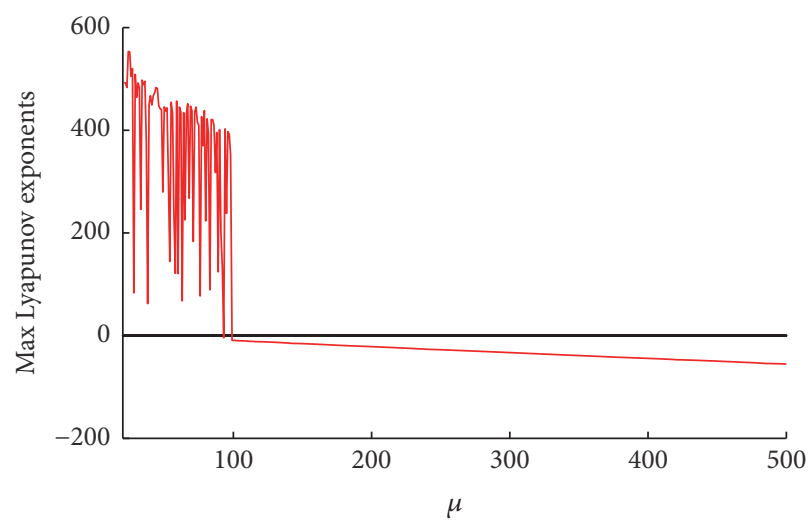

(d)

FIGURE 12: Bifurcation and maximum Lyapunov exponent diagrams with the damping $\mu$, when the imperfection type of the FGM conical panel is $0.1 h \sin (3 \pi x / L)$ : (a) bifurcation diagram of the first mode; (b) bifurcation diagram of the second mode; (c) three-dimensional bifurcation diagram of the first mode; (d) maximum Lyapunov exponent diagram.

$$
\begin{array}{ll}
+\frac{1}{R} A_{66} \frac{\partial^{2} v_{0}}{\partial x^{2}} \frac{\partial w_{0}}{\partial \theta}-\frac{2}{R^{2}} A_{66} \frac{\partial^{2} w_{0}}{\partial x \partial \theta} v_{0} \sin \beta & -\frac{1}{R^{2}} B_{66} \frac{\partial w_{0}}{\partial \theta} \frac{\partial \phi_{\theta}}{\partial x} \sin \beta \\
+\frac{1}{R} A_{12} \frac{\partial^{2} w_{0}}{\partial x^{2}} u_{0} \sin \beta+\frac{1}{R^{3}} A_{66} \frac{\partial w_{0}}{\partial \theta} v_{0} \sin ^{2} \beta & +\frac{1}{R^{3}}\left(A_{22}-A_{66}\right) \frac{\partial w_{0}}{\partial \theta} \frac{\partial u_{0}}{\partial \theta} \sin \beta \\
+\frac{1}{R} B_{66} \frac{\partial^{2} \phi_{\theta}}{\partial x^{2}} \frac{\partial w_{0}}{\partial \theta}+\frac{1}{R^{2}}\left(K A_{44}+N_{\theta \theta}^{T}\right) \frac{\partial^{2} w_{0}}{\partial \theta^{2}} & +\frac{3}{2 R^{4}} A_{22} \frac{\partial^{2} w_{0}}{\partial \theta^{2}}\left(\frac{\partial w_{0}}{\partial \theta}\right)^{2}+\frac{2}{R^{2}} A_{66} \frac{\partial^{2} w_{0}}{\partial x \partial \theta} \frac{\partial u_{0}}{\partial \theta} \\
+\frac{1}{R} A_{12} \frac{\partial^{2} w_{0}}{\partial x^{2}} \frac{\partial v_{0}}{\partial \theta}+\frac{1}{R^{3}} A_{22} \frac{\partial^{2} w_{0}}{\partial \theta^{2}} \frac{\partial v_{0}}{\partial \theta} & +\frac{1}{2 R}\left(A_{12}+2 A_{66}\right) \frac{\partial^{2} w_{0}}{\partial x^{2}}\left(\frac{\partial w_{0}}{\partial \theta}\right)^{2} \\
+K A_{55} \frac{\partial \phi_{x}}{\partial x} \frac{\partial w_{0}}{\partial \theta}-\frac{1}{R^{2}}\left(K A_{44}+A_{22}\right) \frac{\partial v_{0}}{\partial \theta} \cos \beta & +3 A_{11} \frac{\partial^{3} w_{0}}{\partial x^{3}} \frac{\partial w^{*}}{\partial x}+\frac{1}{R} A_{12} \frac{\partial w_{0}}{\partial x} \frac{\partial w^{*}}{\partial x} \cos \beta \\
+B_{11} \frac{\partial^{2} w_{0}}{\partial x^{2}} \frac{\partial \phi_{x}}{\partial x}-\frac{1}{R} B_{12} \frac{\partial \phi_{x}}{\partial x} \cos \beta & +\frac{1}{R^{2}}\left(A_{12}+4 A_{66}\right) \frac{\partial w_{0}}{\partial x \partial \theta} \frac{\partial^{2} w^{*}}{\partial x \partial \theta} \\
+\frac{1}{R^{2}} B_{12} \frac{\partial^{2} w_{0}}{\partial \theta^{2}} \frac{\partial \phi_{x}}{\partial x}+\frac{1}{R^{2}} B_{66} \frac{\partial^{2} \phi_{x}}{\partial \theta^{2}} \frac{\partial w_{0}}{\partial x} & +\frac{1}{R^{2}} A_{12} \frac{\partial^{2} w^{*}}{\partial \theta^{2}} \frac{\partial^{4} w_{0}}{\partial x^{2} \partial \theta^{2}} \\
+\frac{1}{2 R^{3}} A_{22} \frac{\partial^{2} w_{0}}{\partial \theta^{2}} \cos \beta+\frac{2}{R} B_{66} \frac{\partial^{2} w_{0}}{\partial x \partial \theta} \frac{\partial \phi_{\theta}}{\partial x} & +\frac{1}{R^{3}}\left(A_{12}-2 A_{66}\right) \frac{\partial w^{*}}{\partial \theta} \frac{\partial^{2} w_{0}}{\partial x \partial \theta} \sin \beta \\
+\frac{1}{R^{3}} B_{22} \frac{\partial^{2} \phi_{\theta}}{\partial \theta^{2}} \frac{\partial w_{0}}{\partial \theta}+\frac{1}{R^{2}}\left(A_{12}+A_{66}\right) \frac{\partial^{2} u_{0}}{\partial x \partial \theta} \frac{\partial w_{0}}{\partial \theta} & +x^{2}
\end{array}
$$




$$
\begin{aligned}
& +\frac{1}{R^{2}}\left(A_{12}+4 A_{66}\right) \frac{\partial w^{*}}{\partial \theta} \frac{\partial^{3} w_{0}}{\partial x^{2} \partial \theta} \\
& +\frac{1}{R^{3}} A_{22} \frac{\partial w^{*}}{\partial \theta} \frac{\partial^{2} v_{0}}{\partial \theta^{2}}+\frac{1}{R^{3}} A_{22} \frac{\partial^{2} w^{*}}{\partial \theta^{2}} \cos \beta \\
& +\frac{1}{R^{2}}\left(A_{12}+2 A_{66}\right) \frac{\partial w^{*}}{\partial x} \frac{\partial^{3} w_{0}}{\partial x \partial \theta^{2}} \\
& +\frac{1}{R^{3}} B_{22} \frac{\partial w^{*}}{\partial \theta} \frac{\partial^{2} \phi_{\theta}}{\partial \theta^{2}}+A_{11} \frac{\partial w^{*}}{\partial x} \frac{\partial^{2} u_{0}}{\partial x^{2}} \\
& +\frac{1}{R^{2}} A_{12} \frac{\partial^{3} w^{*}}{\partial \theta^{2} \partial x} \frac{\partial^{3} w_{0}}{\partial \theta^{2} \partial x} \\
& +\frac{1}{R} A_{12} \frac{\partial^{2} w^{*}}{\partial x^{2}} \cos \beta+B_{11} \frac{\partial w^{*}}{\partial x} \frac{\partial^{2} \phi_{x}}{\partial x^{2}} \\
& -\frac{2}{R^{3}} A_{12} \frac{\partial^{2} w^{*}}{\partial \theta^{2}} \frac{\partial^{3} w_{0}}{\partial \theta^{2} \partial x} \sin \beta+2 A_{11} \frac{\partial^{3} w^{*}}{\partial x^{3}} \frac{\partial w_{0}}{\partial x} \\
& +\frac{1}{R^{2}} A_{66} \frac{\partial w^{*}}{\partial x} \frac{\partial^{2} u_{0}}{\partial \theta^{2}}+\frac{1}{R} B_{66} \frac{\partial w^{*}}{\partial \theta} \frac{\partial^{2} \phi_{\theta}}{\partial x^{2}} \\
& +\frac{1}{R} K A_{55} \frac{\partial w_{0}}{\partial x} \sin \beta \\
& +\frac{2}{R^{2}}\left(A_{12}+2 A_{66}\right) \frac{\partial^{3} w^{*}}{\partial \theta^{2} \partial x} \frac{\partial w_{0}}{\partial x} \\
& +\frac{1}{R} A_{11} \frac{\partial^{2} w^{*}}{\partial x^{2}} \frac{\partial w_{0}}{\partial x} \sin \beta-\frac{1}{R^{3}} A_{66} \frac{\partial^{2} w^{*}}{\partial \theta^{2}} \frac{\partial w_{0}}{\partial x} \\
& +\frac{1}{R}\left(B_{11}+B_{12}\right) \frac{\partial w^{*}}{\partial x} \frac{\partial \phi_{x}}{\partial x}+\frac{1}{R^{2}} B_{12} \frac{\partial^{2} w^{*}}{\partial \theta^{2}} \frac{\partial \phi_{x}}{\partial x} \\
& +K A_{55} \frac{\partial \phi_{x}}{\partial x}+B_{11} \frac{\partial^{2} w^{*}}{\partial x^{2}} \frac{\partial \phi_{x}}{\partial x}+\frac{3}{2 R} A_{11} \frac{\partial w^{*}}{\partial x} \frac{\partial^{2} w_{0}}{\partial x^{2}} \\
& +\frac{1}{2 R^{2}}\left(A_{12}+2 A_{66}\right) \frac{\partial^{2} w^{*}}{\partial \theta^{2}} \frac{\partial^{2} w_{0}}{\partial x^{2}} \\
& +\frac{3}{2} A_{11} \frac{\partial^{2} w^{*}}{\partial x^{2}} \frac{\partial^{2} w_{0}}{\partial x^{2}} \\
& +\frac{1}{R}\left(A_{11}+A_{12}\right) \frac{\partial w^{*}}{\partial x} \frac{\partial u_{0}}{\partial x} \sin \beta \\
& +\frac{1}{R^{2}} A_{12} \frac{\partial^{2} w^{*}}{\partial \theta^{2}} \frac{\partial u_{0}}{\partial x}+A_{11} \frac{\partial^{2} w^{*}}{\partial x^{2}} \frac{\partial u_{0}}{\partial x} \\
& -\frac{1}{R^{2}} A_{66} \frac{\partial w^{*}}{\partial \theta} \frac{\partial v_{0}}{\partial x} \sin \beta+\frac{2}{R} A_{66} \frac{\partial^{2} w^{*}}{\partial x \partial \theta} \frac{\partial v_{0}}{\partial x} \\
& -\frac{2}{R^{2}} B_{66} \frac{\partial^{2} w^{*}}{\partial x \partial \theta} \phi_{\theta} \sin \beta+\frac{1}{R^{3}} B_{66} \frac{\partial w^{*}}{\partial \theta} \phi_{\theta} \sin ^{2} \beta \\
& +\frac{1}{R} A_{12} w^{*} \frac{\partial^{2} w^{*}}{\partial x^{2}} \cos \beta+\frac{1}{R^{3}} A_{22} w^{*} \frac{\partial^{2} w^{*}}{\partial \theta^{2}} \cos \beta \\
& +\frac{1}{R} K A_{55} \frac{\partial w^{*}}{\partial x} \sin \beta+\frac{1}{R} A_{12} w_{0} \frac{\partial^{2} w^{*}}{\partial x^{2}} \cos \beta \\
& +\frac{1}{R^{3}} A_{22} w_{0} \frac{\partial^{2} w^{*}}{\partial \theta^{2}} \cos \beta+\frac{1}{R} B_{12} \phi_{x} \frac{\partial^{2} w^{*}}{\partial x^{2}} \sin \beta \\
& +\frac{1}{R^{3}} B_{22} \phi_{x} \frac{\partial^{2} w^{*}}{\partial \theta^{2}} \sin \beta+\frac{1}{R} K A_{55} \phi_{x} \sin \beta \\
& +\frac{1}{R^{3}} A_{22} u_{0} \frac{\partial^{2} w^{*}}{\partial \theta^{2}} \sin \beta+\frac{1}{R} A_{12} u_{0} \frac{\partial^{2} w^{*}}{\partial x^{2}} \sin \beta \\
& +\frac{1}{R^{3}} A_{66} v_{0} \frac{\partial w^{*}}{\partial \theta} \sin ^{2} \beta-\frac{2}{R^{2}} A_{66} v_{0} \frac{\partial^{2} w^{*}}{\partial x \partial \theta} \sin \beta \\
& +\frac{1}{R} N_{x x}^{T} \frac{\partial w^{*}}{\partial x} \sin \beta+\frac{1}{R} A_{66} \frac{\partial w^{*}}{\partial \theta} \frac{\partial^{2} v_{0}}{\partial x^{2}} \\
& +K A_{55} \frac{\partial^{2} w^{*}}{\partial x^{2}}+\frac{1}{R^{2}} K A_{44} \frac{\partial^{2} w^{*}}{\partial \theta^{2}} \\
& +\frac{1}{R^{2}} B_{66} \frac{\partial w^{*}}{\partial x} \frac{\partial^{2} \phi_{x}}{\partial \theta^{2}}-\frac{1}{R^{2}} A_{22} w^{*} \cos ^{2} \beta \\
& -\frac{1}{R^{2}} B_{66} \frac{\partial w^{*}}{\partial \theta} \frac{\partial \phi_{\theta}}{\partial x} \sin \beta+\frac{2}{R} B_{66} \frac{\partial^{2} w^{*}}{\partial x \partial \theta} \frac{\partial \phi_{\theta}}{\partial x} \\
& +\frac{1}{R^{2}}\left(B_{12}+B_{66}\right) \frac{\partial w^{*}}{\partial \theta} \frac{\partial^{2} \phi_{x}}{\partial x \partial \theta} \\
& +\frac{1}{R}\left(B_{12}+B_{66}\right) \frac{\partial w^{*}}{\partial x} \frac{\partial^{2} \phi_{\theta}}{\partial x \partial \theta}+\frac{1}{R^{4}} A_{22} \frac{\partial^{2} w^{*}}{\partial \theta^{2}} \frac{\partial^{2} w_{0}}{\partial \theta^{2}} \\
& +\frac{1}{R^{3}} A_{22} w^{*} \frac{\partial^{2} w_{0}}{\partial \theta^{2}} \cos \beta+\frac{1}{R^{2}} A_{66} \frac{\partial^{2} w^{*}}{\partial x^{2}} \frac{\partial^{2} w_{0}}{\partial \theta^{2}} \\
& +\frac{1}{R} A_{12} w^{*} \frac{\partial^{2} w_{0}}{\partial x^{2}} \cos \beta+A_{11} \frac{\partial^{2} w^{*}}{\partial x^{2}} \frac{\partial^{2} w_{0}}{\partial x^{2}} \\
& +\frac{1}{R^{2}}\left(A_{12}+A_{66}\right) \frac{\partial w^{*}}{\partial \theta} \frac{\partial^{2} u_{0}}{\partial x \partial \theta} \\
& +\frac{1}{R^{2}}\left(A_{12}+2 A_{66}\right) \frac{\partial^{2} w^{*}}{\partial x \partial \theta} \frac{\partial^{2} w_{0}}{\partial x \partial \theta} \\
& +\frac{1}{R^{2}}\left(A_{12}+A_{66}\right) \frac{\partial w^{*}}{\partial x} \frac{\partial^{2} v_{0}}{\partial x \partial \theta} \\
& +\frac{1}{R^{2}} A_{66} \frac{\partial^{2} w^{*}}{\partial \theta^{2}} \frac{\partial^{2} w_{0}}{\partial x^{2}}-\frac{1}{R^{2}} B_{66} \frac{\partial w^{*}}{\partial x} \frac{\partial \phi_{\theta}}{\partial \theta} \sin \beta \\
& +\frac{1}{R^{3}} B_{22} \frac{\partial^{2} w^{*}}{\partial \theta^{2}} \frac{\partial \phi_{\theta}}{\partial \theta}+\frac{1}{R} B_{12} \frac{\partial^{2} w^{*}}{\partial x^{2}} \frac{\partial \phi_{\theta}}{\partial \theta} \\
& +\frac{1}{R^{2}}\left(A_{12}+2 A_{66}\right) \frac{\partial w^{*}}{\partial \theta} \frac{\partial^{3} w_{0}}{\partial x^{2} \partial \theta} \\
& +\frac{1}{R^{2}}\left(2 A_{12}+3 A_{66}\right) \frac{\partial w^{*}}{\partial x} \frac{\partial^{3} w_{0}}{\partial x \partial \theta^{2}} \\
& +\frac{1}{R^{2}} A_{12} \frac{\partial^{3} w^{*}}{\partial x^{2} \partial \theta} \frac{\partial w_{0}}{\partial \theta}+\frac{1}{R^{3}} A_{22} \frac{\partial w^{*}}{\partial \theta} \frac{\partial w_{0}}{\partial \theta} \cos \beta \\
& +\frac{3}{R^{4}} A_{22} \frac{\partial w^{*}}{\partial \theta} \frac{\partial^{3} w_{0}}{\partial \theta^{3}}+\frac{4}{R^{2}} A_{66} \frac{\partial^{3} w^{*}}{\partial x^{2} \partial \theta} \frac{\partial w_{0}}{\partial \theta}
\end{aligned}
$$




$$
\begin{aligned}
& +\frac{2}{R^{4}} A_{22} \frac{\partial^{3} w^{*}}{\partial \theta^{3}} \frac{\partial w_{0}}{\partial \theta}+\frac{1}{R^{2}} A_{66} \frac{\partial^{3} w_{0}}{\partial x \partial \theta^{2}} \frac{\partial w^{*}}{\partial x} \\
& -\left(K A_{55}+\frac{1}{R^{2}} D_{22}\right) \phi_{x} \\
& +\frac{1}{R^{3}}\left(A_{12}-A_{66}\right) \frac{\partial^{2} w^{*}}{\partial x \partial \theta} \frac{\partial w_{0}}{\partial \theta} \sin \beta \\
& -\frac{1}{R^{2}}\left(D_{22}+D_{66}\right) \frac{\partial \phi_{\theta}}{\partial \theta} \sin \beta+\frac{1}{R} M_{x x}^{T} \sin \beta \\
& +\frac{1}{R^{2}} A_{12} \frac{\partial^{3} w_{0}}{\partial x \partial \theta^{2}} \frac{\partial^{3} w^{*}}{\partial x \partial \theta^{2}}+\frac{\partial^{2} w^{*}}{\partial x^{2}} N_{x x}^{T} \\
& +\frac{1}{R^{2}} \frac{\partial^{2} w^{*}}{\partial \theta^{2}} N_{\theta \theta}^{T}+\frac{1}{R^{3}}\left(B_{22}-B_{66}\right) \frac{\partial w^{*}}{\partial \theta} \frac{\partial \phi_{x}}{\partial \theta} \sin \beta \\
& +\frac{2}{R^{2}} B_{66} \frac{\partial^{2} w^{*}}{\partial x \partial \theta} \frac{\partial \phi_{x}}{\partial \theta}+\frac{1}{R^{2}} A_{12} \frac{\partial^{4} w^{*}}{\partial x^{2} \partial \theta^{2}} \frac{\partial^{2} w_{0}}{\partial \theta^{2}} \\
& -\frac{2}{R^{3}} A_{12} \frac{\partial^{3} w^{*}}{\partial x \partial \theta^{2}} \frac{\partial^{2} w_{0}}{\partial \theta^{2}} \sin \beta \\
& -\frac{1}{2 R^{3}}\left(A_{12}-2 A_{66}\right) \frac{\partial w^{*}}{\partial x} \frac{\partial^{2} w_{0}}{\partial \theta^{2}} \sin \beta \\
& +\frac{3}{2 R^{4}} A_{22} \frac{\partial^{2} w^{*}}{\partial \theta^{2}} \frac{\partial^{2} w_{0}}{\partial \theta^{2}} \\
& +\frac{1}{2 R^{2}}\left(A_{12}+2 A_{66}\right) \frac{\partial^{2} w^{*}}{\partial x^{2}} \frac{\partial^{2} w_{0}}{\partial \theta^{2}} \\
& +\frac{1}{R^{3}}\left(A_{22}-A_{66}\right) \frac{\partial w^{*}}{\partial \theta} \frac{\partial u_{0}}{\partial \theta} \sin \beta \\
& +\frac{2}{R^{2}} A_{66} \frac{\partial^{2} w^{*}}{\partial x \partial \theta} \frac{\partial u_{0}}{\partial \theta}-\frac{1}{R^{2}} A_{66} \frac{\partial w^{*}}{\partial x} \frac{\partial v_{0}}{\partial \theta} \sin \beta \\
& +\frac{1}{R^{3}} A_{22} \frac{\partial^{2} w^{*}}{\partial \theta^{2}} \frac{\partial v_{0}}{\partial \theta}+\frac{1}{R} A_{12} \frac{\partial^{2} w^{*}}{\partial x^{2}} \frac{\partial v_{0}}{\partial \theta}=I_{0} \ddot{w}_{0}, \\
& B_{11} \frac{\partial^{2} u_{0}}{\partial x^{2}}+\frac{1}{R^{2}} B_{66} \frac{\partial^{2} u_{0}}{\partial \theta^{2}}+\frac{1}{R} B_{11} \frac{\partial u_{0}}{\partial x} \sin \beta \\
& +\frac{1}{R}\left(D_{12}+D_{66}\right) \frac{\partial^{2} \phi_{x}}{\partial x \partial \theta}+\frac{1}{R}\left(B_{12}+B_{66}\right) \frac{\partial^{2} v_{0}}{\partial x \partial \theta} \\
& -\frac{1}{R^{2}} B_{22} u_{0} \sin ^{2} \beta-\frac{1}{R^{2}}\left(B_{22}+B_{66}\right) \frac{\partial v_{0}}{\partial \theta} \sin \beta \\
& +\frac{1}{2 R}\left(B_{11}-B_{12}\right) \frac{\partial^{2} w_{0}}{\partial x^{2}} \sin \beta-K A_{55} \frac{\partial w_{0}}{\partial x} \\
& +\frac{1}{R} B_{12} \frac{\partial w_{0}}{\partial x} \cos \beta+B_{11} \frac{\partial^{2} w_{0}}{\partial x^{2}} \frac{\partial w_{0}}{\partial x} \\
& +\frac{1}{R^{2}} B_{66} \frac{\partial^{2} w_{0}}{\partial \theta^{2}} \frac{\partial w_{0}}{\partial x}+\frac{1}{R^{2}} D_{66} \frac{\partial^{2} \phi_{x}}{\partial \theta^{2}} \\
& +\frac{1}{R} D_{11} \frac{\partial \phi_{x}}{\partial x} \sin \beta-\frac{1}{R^{2}} B_{22} w_{0} \sin \beta \cos \beta \\
& -\frac{1}{2 R^{2}}\left(B_{12}+B_{22}\right) \frac{\partial^{2} w_{0}}{\partial \theta^{2}} \sin \beta+D_{11} \frac{\partial^{2} \phi_{x}}{\partial x^{2}} \\
& +\frac{1}{R^{2}}\left(B_{12}+B_{66}\right) \frac{\partial^{2} w_{0}}{\partial x \partial \theta} \frac{\partial w_{0}}{\partial \theta} \\
& \frac{1}{R}\left(B_{12}+B_{66}\right) \frac{\partial^{2} u_{0}}{\partial x \partial \theta}+\frac{1}{R^{2}}\left(B_{22}+B_{66}\right) \frac{\partial u_{0}}{\partial \theta} \sin \beta \\
& +\left(\frac{1}{R} K A_{44} \cos \beta-\frac{1}{R^{2}} B_{66} \sin ^{2} \beta\right) v_{0}+B_{66} \frac{\partial^{2} v_{0}}{\partial x^{2}} \\
& +\frac{1}{R^{2}} B_{22} \frac{\partial^{2} v_{0}}{\partial \theta^{2}}+\frac{1}{R^{2}}\left(D_{22}+D_{66}\right) \frac{\partial \phi_{\theta}}{\partial \theta} \\
& +\frac{1}{R^{2}} B_{66} \frac{\partial w_{0}}{\partial x} \frac{\partial w_{0}}{\partial \theta} \sin \beta \\
& +\left(\frac{1}{R^{2}} B_{22} \cos \beta-\frac{1}{R} K A_{44}\right) \frac{\partial w_{0}}{\partial \theta} \\
& +\frac{1}{R^{3}} B_{22} \frac{\partial^{2} w_{0}}{\partial \theta^{2}} \frac{\partial w_{0}}{\partial \theta}+\frac{1}{R} B_{66} \frac{\partial^{2} w_{0}}{\partial x^{2}} \frac{\partial w_{0}}{\partial \theta} \\
& +\frac{1}{R^{2}} D_{22} \frac{\partial^{2} \phi_{\theta}}{\partial \theta^{2}}-\left(K A_{44}+\frac{1}{R^{2}} D_{66} \sin ^{2} \beta\right) \phi_{\theta} \\
& +\frac{1}{R} B_{66} \frac{\partial v_{0}}{\partial x} \sin \beta+\frac{1}{R}\left(B_{12}+B_{66}\right) \frac{\partial^{2} w_{0}}{\partial x \partial \theta} \frac{\partial w_{0}}{\partial x} \\
& +\frac{1}{R}\left(D_{12}+D_{66}\right) \frac{\partial^{2} \phi_{x}}{\partial x \partial \theta}+D_{66} \frac{\partial^{2} \phi_{\theta}}{\partial x^{2}}+\frac{1}{R} D_{66} \frac{\partial \phi_{\theta}}{\partial x} \\
& +\frac{1}{R^{2}} B_{66} \frac{\partial w^{*}}{\partial \theta} \frac{\partial w_{0}}{\partial x} \sin \beta
\end{aligned}
$$




$$
\begin{aligned}
& +\frac{1}{R}\left(B_{12}+B_{66}\right) \frac{\partial^{2} w^{*}}{\partial x \partial \theta} \frac{\partial w_{0}}{\partial x} \\
& +\frac{1}{R^{2}} B_{66} \frac{\partial w^{*}}{\partial x} \frac{\partial w_{0}}{\partial \theta} \sin \beta+\frac{1}{R^{3}} B_{22} \frac{\partial^{2} w^{*}}{\partial \theta^{2}} \frac{\partial w_{0}}{\partial \theta} \\
& +\frac{1}{R} B_{66} \frac{\partial^{2} w^{*}}{\partial x^{2}} \frac{\partial w_{0}}{\partial \theta}+\frac{1}{R} B_{66} \frac{\partial^{2} w_{0}}{\partial x^{2}} \frac{\partial w^{*}}{\partial \theta} \\
& +\frac{1}{R}\left(B_{12}+B_{66}\right) \frac{\partial w^{*}}{\partial x} \frac{\partial^{2} w_{0}}{\partial x \partial \theta}+\frac{1}{R^{2}} B_{22} \frac{\partial w^{*}}{\partial \theta} \cos \beta \\
& -\frac{1}{R} K A_{44} \frac{\partial w^{*}}{\partial \theta}+\frac{1}{R^{3}} B_{22} \frac{\partial^{2} w_{0}}{\partial \theta^{2}} \frac{\partial w^{*}}{\partial \theta}=I_{1} \ddot{v}_{0} \\
& +I_{2} \ddot{\phi}_{\theta} .
\end{aligned}
$$

\section{Conflicts of Interest}

The authors declare that there are no conflicts of interest regarding the publication of this paper.

\section{Acknowledgments}

The authors gratefully acknowledge the support of the National Natural Science Foundation of China (NNSFC) through Grant nos. 11372015 and 11272063 and the ASME conference.

\section{References}

[1] M. M. Aghdam, N. Shahmansouri, and K. Bigdeli, "Bending analysis of moderately thick functionally graded conical panels," Composite Structures, vol. 93, no. 5, pp. 1376-1384, 2011.

[2] J. Abediokhchi, M. Shakouri, and M. A. Kouchakzadeh, "Bending analysis of moderately thick functionally graded conical panels with various boundary conditions using GDQ method," Composite Structures, vol. 103, pp. 68-74, 2013.

[3] M. M. Aghdam, N. Shahmansouri, and M. Mohammadi, "Extended Kantorovich method for static analysis of moderately thick functionally graded sector plates," Mathematics and Computers in Simulation, vol. 86, pp. 118-130, 2012.

[4] N. D. Duc, P. H. Cong, V. M. Anh et al., "Mechanical and thermal stability of eccentrically stiffened functionally graded conical shell panels resting on elastic foundations and in thermal environment," Composite Structures, vol. 132, pp. 597-609, 2015.

[5] E. Viola, L. Rossetti, N. Fantuzzi, and F. Tornabene, "Static analysis of functionally graded conical shells and panels using the generalized unconstrained third order theory coupled with the stress recovery," Composite Structures, vol. 112, no. 1, pp. 44-65, 2014.

[6] N. S. Bardell, R. S. Langley, J. M. Dunsdon, and G. S. Aglietti, "An h-p finite element vibration analysis of open conical sandwich panels and conical sandwich frusta," Journal of Sound and Vibration, vol. 226, no. 2, pp. 345-377, 1999.

[7] F. Tornabene and E. Viola, "Free vibrations of four-parameter functionally graded parabolic panels and shells of revolution," European Journal of Mechanics - A/Solids, vol. 28, no. 5, pp. 9911013, 2009.
[8] X. Zhao and K. M. Liew, "Free vibration analysis of functionally graded conical shell panels by a meshless method," Composite Structures, vol. 93, no. 2, pp. 649-664, 2011.

[9] M. Akbari, Y. Kiani, M. M. Aghdam, and M. R. Eslami, "Free vibration of FGM Lévy conical panels," Composite Structures, vol. 116, no. 1, pp. 732-746, 2014.

[10] Z. Su, G. Jin, S. Shi, T. Ye, and X. Jia, "A unified solution for vibration analysis of functionally graded cylindrical, conical shells and annular plates with general boundary conditions," International Journal of Mechanical Sciences, vol. 80, pp. 62-80, 2014.

[11] Z. Su, G. Jin, and X. Wang, "Free vibration analysis of laminated composite and functionally graded sector plates with general boundary conditions," Composite Structures, vol. 132, pp. 720736, 2015.

[12] X. Xie, G. Jin, T. Ye, and Z. Liu, "Free vibration analysis of functionally graded conical shells and annular plates using the Haar wavelet method," Applied Acoustics, vol. 85, pp. 130-142, 2014.

[13] F. Tornabene, "Free vibration analysis of functionally graded conical, cylindrical shell and annular plate structures with a four-parameter power-law distribution," Computer Methods Applied Mechanics and Engineering, vol. 198, no. 37-40, pp. 29112935, 2009.

[14] G. Hafeez, A. M. El Ansary, and A. A. El Damatty, "Stability of combined imperfect conical tanks under hydrostatic loading," Journal of Constructional Steel Research, vol. 66, no. 11, pp. 13871397, 2010.

[15] M. Maali, H. Showkati, and S. Mahdi Fatemi, "Investigation of the buckling behavior of conical shells under weld-induced imperfections," Thin-Walled Structures, vol. 57, pp. 13-24, 2012.

[16] X. Zhao and K. M. Liew, "An element-free analysis of mechanical and thermal buckling of functionally graded conical shell panels," International Journal for Numerical Methods in Engineering, vol. 86, no. 3, pp. 269-285, 2011.

[17] D. H. Bich, N. T. Phuong, and H. V. Tung, "Buckling of functionally graded conical panels under mechanical loads," Composite Structures, vol. 94, no. 4, pp. 1379-1384, 2012.

[18] M. Javanbakht, M. Shakeri, S. N. Sadeghi, and A. R. Daneshmehr, "The analysis of functionally graded shallow and nonshallow shell panels with piezoelectric layers under dynamic load and electrostatic excitation based on elasticity," European Journal of Mechanics - A/Solids, vol. 30, no. 6, pp. 983-991, 2011.

[19] C.-P. Fung and C.-S. Chen, "Imperfection sensitivity in the nonlinear vibration of functionally graded plates," European Journal of Mechanics - A/Solids, vol. 25, no. 3, pp. 425-436, 2006.

[20] M. Ghadimi, M. Dardel, M. H. Pashaei, and M. M. Barzegari, "Effects of geometric imperfections on the aeroelastic behavior of functionally graded wings in supersonic flow," Aerospace Science and Technology, vol. 23, no. 1, pp. 492-504, 2012.

[21] H. Huang and Q. Han, "Buckling of imperfect functionally graded cylindrical shells under axial compression," European Journal of Mechanics - A/Solids, vol. 27, no. 6, pp. 1026-1036, 2008.

[22] A. H. Sofiyev, "Influence of the initial imperfection on the nonlinear buckling response of FGM truncated conical shells," International Journal of Mechanical Sciences, vol. 53, no. 9, pp. 753-761, 2011.

[23] M. Shariyat and D. Asgari, "Nonlinear thermal buckling and postbuckling analyses of imperfect variable thickness temperature-dependent bidirectional functionally graded cylindrical shells," International Journal of Pressure Vessels and Piping, vol. 111-112, pp. 310-320, 2013. 
[24] B. A. Samsam Shariat and M. R. Eslami, "Thermal buckling of imperfect functionally graded plates," International Journal of Solids and Structures, vol. 43, no. 14-15, pp. 4082-4096, 2006.

[25] J. Yang and X.-L. Huang, "Nonlinear transient response of functionally graded plates with general imperfections in thermal environments," Computer Methods Applied Mechanics and Engineering, vol. 196, no. 25-28, pp. 2619-2630, 2007.

[26] S. Kitipornchai, J. Yang, and K. M. Liew, "Semi-analytical solution for nonlinear vibration of laminated FGM plates with geometric imperfections," International Journal of Solids and Structures, vol. 41, no. 9-10, pp. 2235-2257, 2004.

[27] N. D. Duc and T. Q. Quan, "Nonlinear response of imperfect eccentrically stiffened FGM cylindrical panels on elastic foundation subjected to mechanical loads," European Journal of Mechanics - A/Solids, vol. 46, pp. 60-71, 2014.

[28] Y. Z. Liu, Y. X. Hao, W. Zhang, J. Chen, and S. B. Li, "Nonlinear dynamics of initially imperfect functionally graded circular cylindrical shell under complex loads," Journal of Sound and Vibration, vol. 348, pp. 294-328, 2015.

[29] M. H. Yao, Y. Niu, and W. Zhang, "Nonlinear Dynamics of FGM Conical Panel With Initial Imperfection in Thermal Environment," in Proceedings of the ASME 2017 International Design Engineering Technical Conferences and Computers and Information in Engineering Conference, Cleveland, OH, USA, August 2017.

[30] S. W. Yang, Y. X. Hao, W. Zhang, and S. B. Li, "Nonlinear dynamic behavior of functionally graded truncated conical shell under complex loads," International Journal of Bifurcation and Chaos, vol. 25, no. 2, article 1550025, 2015.

[31] A. Nosier and J. N. Reddy, "A study of non-linear dynamic equations of higher-order shear deformation plate theories," International Journal of Non-Linear Mechanics, vol. 26, no. 2, pp. 233-249, 1991.

[32] A. Bhimaraddi, "Large amplitude vibrations of imperfect antisymmetric angle-ply laminated plates," Journal of Sound and Vibration, vol. 162, no. 3, pp. 457-470, 1993.

[33] Y. X. Hao, W. Zhang, J. Yang, and S. B. Li, "Nonlinear dynamics of a functionally graded thin simply-supported plate under a hypersonic flow," Mechanics of Advanced Materials and Structures, vol. 22, no. 8, pp. 619-632, 2015.

[34] H.-S. Shen and H. Wang, "Nonlinear vibration of shear deformable FGM cylindrical panels resting on elastic foundations in thermal environments," Composites Part B: Engineering, vol. 60, pp. 167-177, 2014.

[35] N. Jooybar, P. Malekzadeh, A. Fiouz, and M. Vaghefi, “Thermal effect on free vibration of functionally graded truncated conical shell panels," Thin-Walled Structures, vol. 103, pp. 45-61, 2016. 


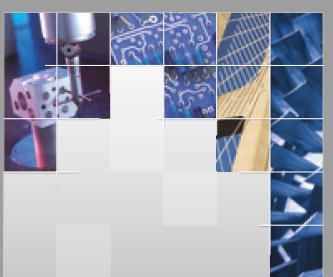

\section{Enfincering}
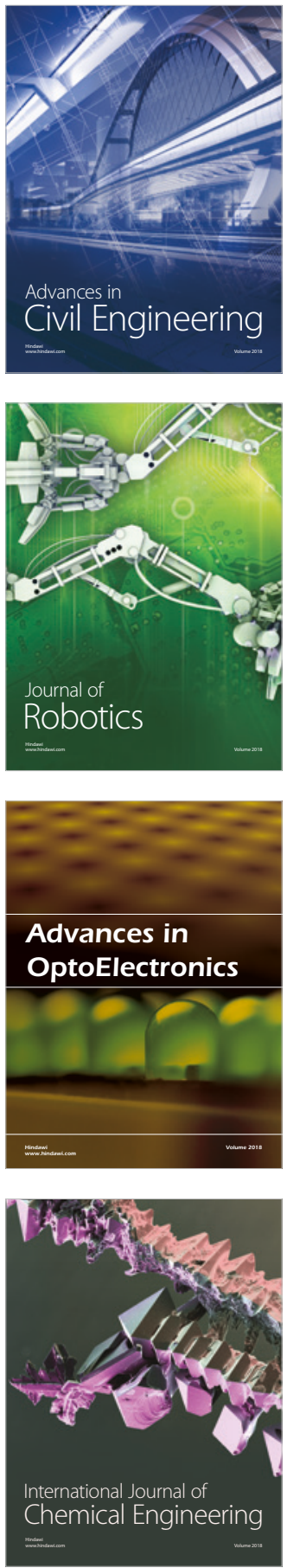

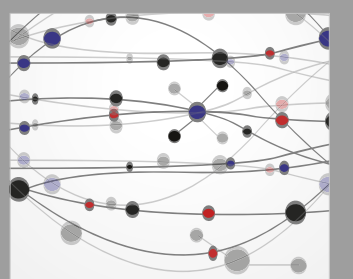

\section{Rotating \\ Machinery}

The Scientific World Journal

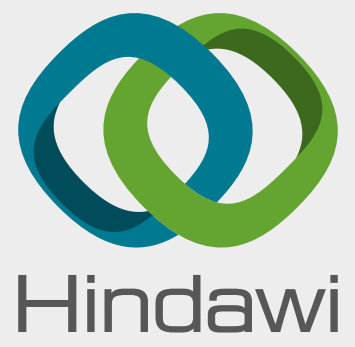

Submit your manuscripts at

www.hindawi.com
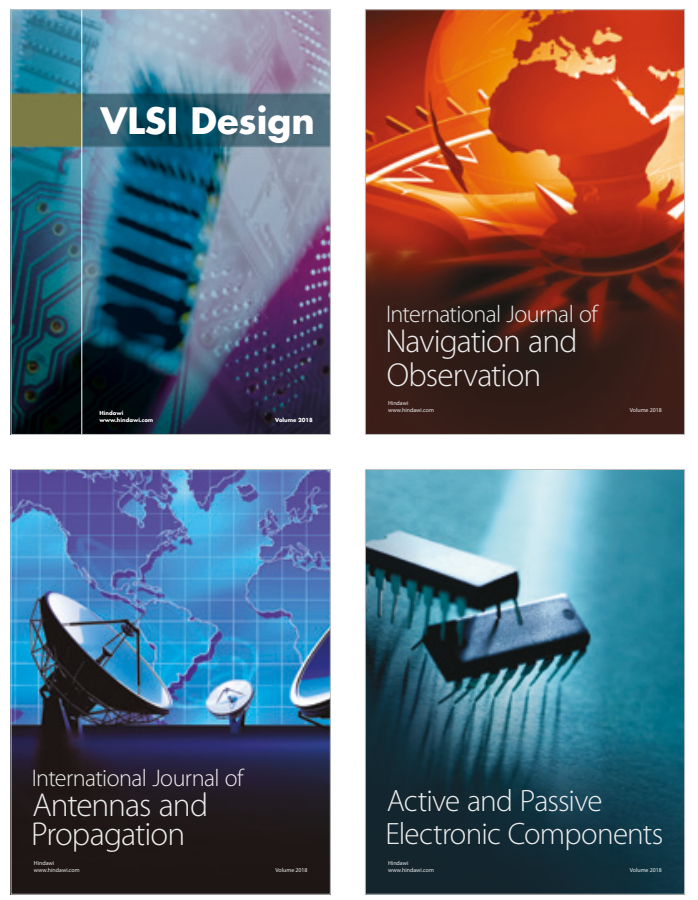
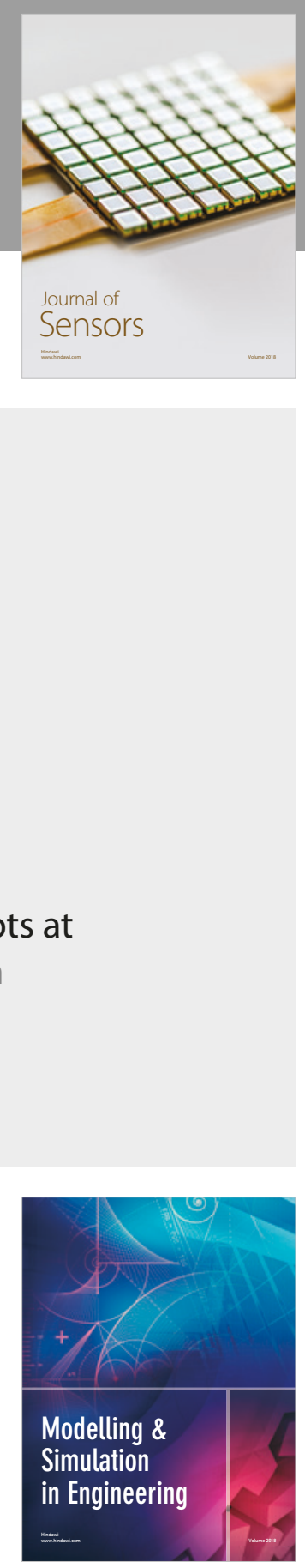

\section{Advances \\ Multimedia}
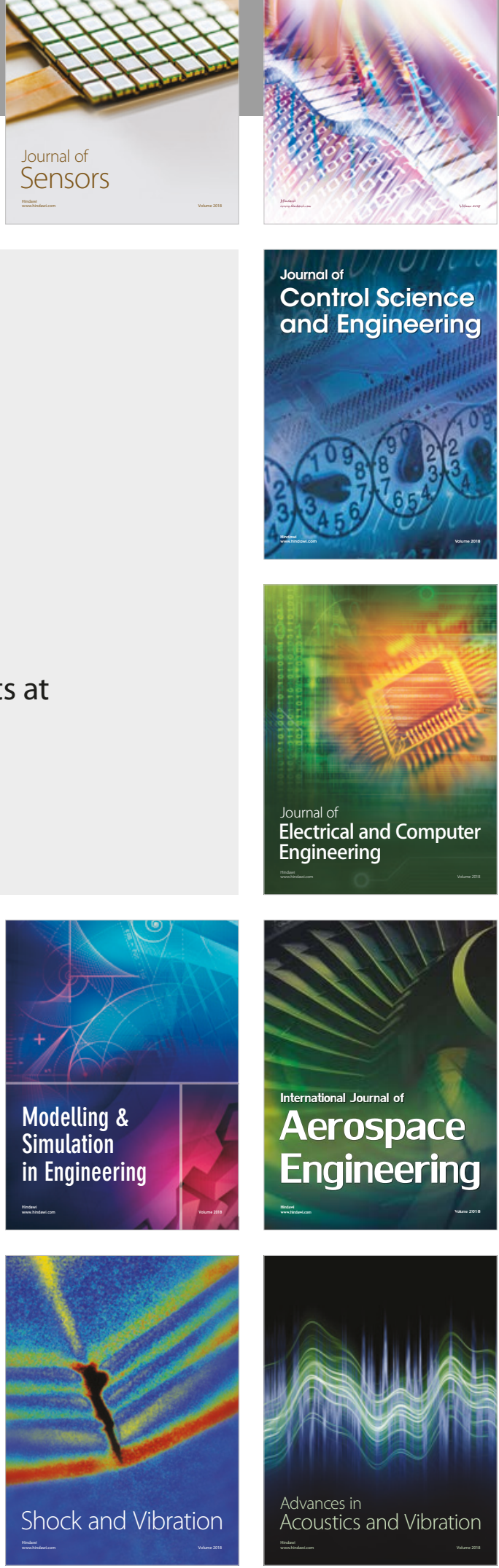